\title{
Multiple Dileucine-like Motifs Direct VGLUT1 Trafficking
}

\author{
Sarah M. Foss, ${ }^{1,4}$ Haiyan Li, ${ }^{1}$ Magda S. Santos, ${ }^{1}$ Robert H. Edwards, ${ }^{2,3}$ and Susan M. Voglmaier ${ }^{1}$ \\ Departments of ${ }^{1}$ Psychiatry, ${ }^{2}$ Neurology, and ${ }^{3}$ Physiology, and ${ }^{4}$ Graduate Program in Cell Biology, School of Medicine, University of California, San \\ Francisco, San Francisco, California 94143-0984
}

The vesicular glutamate transporters (VGLUTs) package glutamate into synaptic vesicles, and the two principal isoforms VGLUT1 and VGLUT2 have been suggested to influence the properties of release. To understand how a VGLUT isoform might influence transmitter release, we have studied their trafficking and previously identified a dileucine-like endocytic motif in the C terminus of VGLUT1. Disruption of this motif impairs the activity-dependent recycling of VGLUT1, but does not eliminate its endocytosis. We now report the identification of two additional dileucine-like motifs in the N terminus of VGLUT1 that are not well conserved in the other isoforms. In the absence of all three motifs, rat VGLUT1 shows limited accumulation at synaptic sites and no longer responds to stimulation. In addition, shRNA-mediated knockdown of clathrin adaptor proteins AP-1 and AP-2 shows that the C-terminal motif acts largely via AP-2, whereas the N-terminal motifs use AP-1. Without the C-terminal motif, knockdown of AP-1 reduces the proportion of VGLUT1 that responds to stimulation. VGLUT1 thus contains multiple sorting signals that engage distinct trafficking mechanisms. In contrast to VGLUT1, the trafficking of VGLUT2 depends almost entirely on the conserved C-terminal dileucine-like motif: without this motif, a substantial fraction of VGLUT2 redistributes to the plasma membrane and the transporter's synaptic localization is disrupted. Consistent with these differences in trafficking signals, wild-type VGLUT1 and VGLUT2 differ in their response to stimulation.

\section{Introduction}

The regulated release of neurotransmitter depends on the assembly into synaptic vesicles of membrane proteins that confer regulated exocytotic release. In addition to proteins that mediate membrane fusion, this process requires trafficking of vesicular transporters necessary to fill synaptic vesicles with transmitter. For glutamate, three distinct vesicular glutamate transporters (VGLUT1, VGLUT2, and VGLUT3) have been identified (Aihara et al., 2000; Bellocchio et al., 2000; Takamori et al., 2000, 2001; Bai et al., 2001; Fremeau et al., 2001, 2002; Herzog et al., 2001; Gras et al., 2002; Schäfer et al., 2002; Takamori et al., 2002). The three isoforms exhibit similar transport activity in vitro, but display mutually exclusive expression patterns in the nervous system (Fremeau et al., 2004a). Surprisingly, the complementary expression of VGLUT1 and VGLUT2 correlates with the properties of transmitter release. VGLUT1 is expressed by neurons in the hippocampus, cerebral cortex, and cerebellar cortex that form synapses with a low probability of vesicle release and high potential for plasticity. In contrast, VGLUT2 is mainly expressed by neurons in the thalamus, brainstem, and deep cerebellar nuclei that

Received Dec. 11, 2012; revised April 29, 2013; accepted May 13, 2013.

Author contributions: S.M.F., H.L., M.S.S., R.H.E., and S.M.V. designed research; S.M.F., H.L., and M.S.S. performed research; S.M.F., H.L., and M.S.S. analyzed data; S.M.F., R.H.E., and S.M.V. wrote the paper.

This work was supported by an NIMH predoctoral fellowship (S.M.F.), grants from NIMH (R.H.E., S.M.V.), and the International Mental Health Research Organization, the Whitehall Foundation, the Brain and Behavior Research Foundation (National Alliance for Research on Schizophrenia and Depression), and the University of California, San Francisco Office of the Dean and the Program for Breakthrough Biomedical Research (S.M.V).

The authors declare no competing financial interests.

Correspondence should be addressed to Dr. Susan M. Voglmaier, Department of Psychiatry, University of California, San Francisco School of Medicine, 401 Parnassus Avenue, LPPI-A101, San Francisco, CA 941430984. E-mail: susan.voglmaier@ucsf.edu.

DOI:10.1523/JNEUROSCI.5662-12.2013

Copyright $\odot 2013$ the authors $\quad 0270-6474 / 13 / 3310647-14 \$ 15.00 / 0$ release transmitter with a higher probability of release at synapses with a lower potential for plasticity (Fremeau et al., 2004a; Omote et al., 2011). Although these observations originally suggested only a correlation, previous work raises the possibility that VGLUT1 and VGLUT2 can control release probability (Weston et al., 2011). One corollary of this hypothesis is that the two isoforms differ in their membrane trafficking.

VGLUTs exhibit a high level of sequence homology in the transmembrane segments that mediate glutamate transport, but diverge considerably at the cytoplasmic $\mathrm{N}$ and $\mathrm{C}$ termini that presumably control intracellular trafficking (Takamori, 2006). The $\mathrm{C}$ terminus of all three isoforms contains a conserved dileucine-like motif that we previously found important for VGLUT1 endocytosis (Voglmaier et al., 2006). In addition, VGLUT1 contains two polyproline domains distal to the C-terminal dileucine-like motif, including one that interacts with the endocytic BAR (Bin/Amphiphysin/Rvs) domain protein endophilin (De Gois et al., 2006; Vinatier et al., 2006; Voglmaier et al., 2006). Endophilin recruits VGLUT1 into the faster AP-2dependent recycling pathway, away from a slower pathway sensitive to brefeldin A, an inhibitor of the AP- 1 and AP-3 adaptors (Voglmaier et al., 2006). Since VGLUT2 and VGLUT3 lack this polyproline motif, the interaction with endophilin may contribute to differences between the isoforms. Previous work has also suggested that interaction with endophilin contributes to the differences in release probability observed between VGLUT1 ${ }^{+}$and $\mathrm{VGLUT}^{+}{ }^{+}$terminals, although the mechanism remains unclear (Weston et al., 2011). The role of endophilin in VGLUT1 trafficking also depends on the upstream dileucine-like motif (Voglmaier et al., 2006).

Although mutagenesis of Phe-510 and Val-511 in the conserved C-terminal dileucine-like motif of VGLUT1 (FV/GG 
VGLUT1) impairs endocytosis, the mutant transporter still undergoes activity-dependent exocytosis and recycling (Voglmaier et al., 2006). We now report the identification of two additional endocytic motifs in VGLUT1 that are not well conserved in VGLUT2, which may contribute to differences observed in trafficking of the two isoforms.

\section{Materials and Methods}

Molecular biology. Standard PCR-directed mutagenesis techniques were used to modify VGLUT1-pH in the pCAGGS vector (Voglmaier et al., 2006). To generate the FV/GG $\Delta \mathrm{PP}$ mutant, a stop codon was introduced after Glu-529 to delete the distal C terminus of VGLUT1-pH (containing both polyproline sequences) and was combined with the FV/GG mutation using standard subcloning techniques. $\Delta$ C-term VGLUT1-pH was generated by replacement of Ser-504 with a stop codon. $\Delta \mathrm{N}$-term VGLUT1-pH was created by PCR mutagenesis, deleting the first 60 aa of VGLUT1 and inserting a new start codon followed by a glutamate residue to preserve the complete Kozak sequence (Fig. 1, N-term start B) (Kozak, 1986). To generate the double truncation mutant, $\Delta \mathrm{N}$-term \& $\Delta \mathrm{C}$-term VGLUT1-pH, the first 50 aa of VGLUT1 were deleted using PCR mutagenesis, inserting a new start codon (Fig. 1, N-term start A) along with a glutamate residue (to preserve the Kozak sequence), and Ser-504 was replaced by a stop codon. A partial amino acid sequence of VGLUT1, with the sites of mutations, truncations, and predicted transmembrane regions indicated, is shown in Figure 1. VGLUT2-pH was constructed in a manner similar to VGLUT1-pH with ecliptic pHluorin inserted in the first luminal loop between Gly-107 and Gly-108 of rat VGLUT2 flanked by the same linker sequences used in VGLUT1-pH (Voglmaier et al., 2006). Tac chimeras were produced by fusing to the $\mathrm{C}$ terminus of Tac either the entire cytoplasmic $\mathrm{C}$ terminus of VGLUT1 beginning with Glu-493 or the N terminus of VGLUT1 from Met-1 to Phe-57, with indicated point mutations. Tac, Tac-VGLUT1 C-terminal, and Tac-VMAT2 constructs have been described previously (Tan et al., 1998; Voglmaier et al., 2006).

RNA interference knockdown. Lentiviral constructs expressing shRNA to rat AP-1 $\gamma$ (AP-1A shRNA, 5'-ACCGAATTAAGAAAGTAGT-3'; AP-1B shRNA, $5^{\prime}$-GGAATGCTATTCTGTATGA- $\left.3^{\prime}\right)$ or AP- $2 \mu$ ( $5^{\prime}$-GT GGATGCCTTTCGCGTCA-3') (Dugast et al., 2005; Kim and Ryan, 2009; Cheung and Cousin, 2012) were made using standard molecular biology procedures in the pFHUBW vector, a variant of pFHUGW containing the monomeric blue fluorescent protein mTagBFP in place of GFP (Lois et al., 2002). The resulting pFHUBW transfer vectors were cotransfected along with two packaging plasmids (pVSV-G and psPAX2) into HEK293T cells using FuGENE HD (Promega). Cells were transfected and grown in UltraCULTURE serum-free media (Lonza) supplemented with $1 \mathrm{~mm}$ sodium pyruvate, $0.075 \%$ sodium bicarbonate, and 2 mM GlutaMax. Approximately $16 \mathrm{~h}$ after transfection, $10 \mu \mathrm{M}$ sodium butyrate was added to the culture media. Approximately $40 \mathrm{~h}$ after transfection, the culture media was collected, and viral particles concentrated by centrifugation through a $20 \%$ sucrose/PBS cushion at $80,000 \times g$ for $2 \mathrm{~h}$. Viral particles were resuspended in neuronal culture media supplemented with $4 \mu \mathrm{g} / \mathrm{ml}$ Polybrene (hexadimethrine bromide) (Zhang et al., 2010).

To confirm specific knockdown, rat hippocampal neurons were infected at $7 \mathrm{~d}$ in vitro (DIV7) and harvested at DIV14. Cells were lysed in $100 \mathrm{~mm}$ Tris- $\mathrm{HCl}, \mathrm{pH}$ 7.5, $150 \mathrm{~mm} \mathrm{NaCl}, 1 \mathrm{~mm}$ EGTA, and 1\% Triton $\mathrm{X}-100$ containing protease inhibitors $(1 \mathrm{mg} / \mathrm{ml} \mathrm{E} 64,2 \mathrm{mg} / \mathrm{ml}$ aprotinin, 2 $\mathrm{mg} / \mathrm{ml}$ leupeptin, $2 \mathrm{mg} / \mathrm{ml}$ pepstatin, and $20 \mathrm{mg} / \mathrm{ml} \mathrm{PMSF}$ ) and centrifuged at $2500 \times g$ for $5 \mathrm{~min}$ to produce a postnuclear supernatant. For immunoblotting, $\sim 25 \mu \mathrm{g}$ of protein was separated by SDS-PAGE and transferred to PVDF membrane (Immobilon-P; Millipore). AP-1, AP-2, and AP-3 were detected by anti-adaptin $\gamma$ (1:500; BD Biosciences), antiadaptin $\alpha$ (1:1000; BD Biosciences), or anti-adaptin $\delta$ (1:250; Developmental Studies Hybridoma Bank), along with appropriate secondary antibodies conjugated to HRP (Jackson ImmunoResearch). Quantifications were performed with ImageJ (http://rsbweb.nih.gov/ij/) using antitubulin (1:3000; EMD Millipore) to normalize the signal. To confirm AP-1 knockdown, selected coverslips with neurons infected with either AP- 1 or vector-only lentiviruses were fixed with 4\% PFA in PBS for 20 min after live imaging. Cells were permeabilized and blocked in PBS containing $1 \%$ fish skin gelatin, $5 \%$ bovine serum albumin, and $0.02 \%$ saponin. AP-1 was then detected using anti-adaptin $\gamma(1: 200$; BD Biosciences) and appropriate secondary antibody.

Internalization of Tac chimeras. Tac chimeras were transfected into HeLa cells using FuGENE HD (Promega). Approximately $48 \mathrm{~h}$ after transfection, cells were transferred to growth media supplemented with $20 \mathrm{~mm}$ HEPES and placed on ice for $15 \mathrm{~min}$ to stop protein trafficking, before incubation with mouse monoclonal anti-Tac antibodies (1:200; Covance) for $30 \mathrm{~min}$ on ice in growth media. To assess antibody uptake, cells were rinsed twice to remove excess antibody, incubated at $37^{\circ} \mathrm{C}$ for $1 \mathrm{~h}$, fixed with $4 \%$ paraformaldehyde in PBS, and blocked with 5\% goat serum in PBS without detergent. To measure surface Tac, intact cells were immunostained with FITC-conjugated anti-mouse antibodies (Jackson ImmunoResearch). To visualize total Tac, cells were then permeabilized with $0.1 \%$ Triton X-100 in PBS containing 5\% goat serum, subsequently stained with Cy3-conjugated anti-mouse antibodies (Jackson ImmunoResearch) and imaged by epifluorescence microscopy (Tan et al., 1998; Voglmaier et al., 2006; Zhao et al., 2008). To quantify the extent of internalization, the degree of colocalization between the surface (green) and total (red) staining for $>60$ cells from two independent transfections per construct was measured using the Correlation Plot function of MetaMorph (Molecular Devices), and the resulting data presented as the average correlation coefficient $\left(r_{\text {avg }}\right)$ (He et al., 2005; Das and Banker, 2006).

Neuronal culture and live cell imaging. Primary hippocampal neurons were cultured as described previously (Li et al., 2011). Briefly, hippocampi were dissected from embryonic day 19 (E19) or E20 rat pups of either sex, dissociated with trypsin, and transfected using the Basic Neuron SCN Nucleofector kit, according to manufacturer's instructions 
(Lonza). Cells were maintained in Neurobasal media supplemented with $1 \%$ heat-inactivated fetal bovine serum, $2 \%$ NeuroMix growth supplement (PAA Laboratories), 2 mm GlutaMax, $15 \mathrm{~mm} \mathrm{NaCl}$, and $10 \mu \mathrm{g} / \mathrm{ml}$ Primocin (Lonza). 5-Fluoro-2'-deoxyuridine and uridine were added at DIV4-DIV6 to a final concentration of $10 \mu \mathrm{M}$ to limit glial growth. Cells were imaged at DIV14-DIV20 in modified Tyrode's solution [containing the following (in mM): $126.5 \mathrm{NaCl}, 30$ dextrose, $2.5 \mathrm{KCl}, 2 \mathrm{CaCl}_{2}, 2$ $\mathrm{MgCl}_{2}$, and $10 \mathrm{HEPES}$ ] containing $10 \mu \mathrm{M}$ each of glutamate receptor antagonists CNQX (6-cyano-7-nitroquinoxaline-2,3-dione) and CPP [3-(2-carboxypiperazin-4-yl)propyl-1-phosphonic acid] (Tocris Bioscience). The total pool size was determined using Tyrode's solution with 50 $\mathrm{mM} \mathrm{NH}_{4} \mathrm{Cl}$ ( $\mathrm{NaCl}$ reduced by $50 \mathrm{~mm}$ to compensate). For knockdown experiments, cells were infected at DIV7 and imaged at DIV14-DIV16.

Cells were imaged as described previously (Voglmaier et al., 2006; Li et al., 2011). Coverslips with transfected neurons were mounted in a perfusion and stimulation chamber (Warner Instruments) on an inverted microscope (Nikon TE3000) fitted with a $63 \times$ oil objective. Cells were imaged in modified Tyrode's solution at room temperature with field stimulation $(5-10 \mathrm{~V} / \mathrm{cm}$ ) applied using $1 \mathrm{~ms}$ bipolar current pulses (A310 Accupulser; WPI). Cells were illuminated using a Xenon lamp (Sutter Instruments) with a $470 / 40 \mathrm{~nm}$ excitation filter and a 525/50 nm emission filter. Images were acquired every $3 \mathrm{~s}$ using a QuantEM CCD camera (Photometrics). MetaMorph software was used to control data collection and to perform offline analysis. All animal work was conducted under the supervision of the Institutional Animal Care and Use Committee of the University of California, San Francisco. Unless otherwise noted, all chemicals were from Sigma, and all tissue culture media were from Life Technologies.

Data analysis. Using MetaMorph software, the average fluorescence of regions of interest at synaptic sites was measured at manually selected $4 \times$ 4 pixel boxes placed over the center of boutons. The average fluorescence of three $4 \times 4$ pixel boxes without cellular elements was subtracted as background. To determine the time course of fluorescence response to stimulation, baseline values from the five frames before stimulation were averaged, and the dynamics of fluorescence intensity expressed as fractional change $(\Delta F)$ over initial fluorescence $\left(F_{0}\right)$. The data were then normalized to either the peak fluorescence in each trace or the total amount of fluorescence as determined by application of modified Tyrode's solution containing $50 \mathrm{mM} \mathrm{NH}_{4} \mathrm{Cl}$ at $\mathrm{pH} 7.4$ to alkalinize all synaptic compartments. To determine the percentage of decline from peak during stimulation, the fluorescence recorded at the last time point of stimulation (60 s) was subtracted from peak fluorescence, and the difference expressed as a percentage of peak fluorescence.

To measure the rate of exocytosis and to determine the total amount of transporter that underwent exocytosis, cells were imaged in modified Tyrode's medium containing 0.5-1 $\mu \mathrm{M}$ bafilomycin A (EMD Millipore). The fraction of transporter that undergoes exocytosis was measured as a fraction of the total internal pool, as determined by subtraction of initial fluorescence from fluorescence in the presence of $50 \mathrm{mM} \mathrm{NH}_{4} \mathrm{Cl}$ (Hua et al., 2011). The size of the readily releasable pool (RRP) was measured with a $100 \mathrm{~Hz}, 0.2 \mathrm{~s}$ stimulus, followed $1 \mathrm{~min}$ later by a $10 \mathrm{~Hz}, 120 \mathrm{~s}$ stimulus to determine the size of the recycling pool (RP) (Ariel and Ryan, 2010). The RRP was measured in modified Tyrode's solution with $\mathrm{CaCl}_{2}$ increased to $4 \mathrm{~mm}$. The bath media was replaced with Tyrode's solution containing $2 \mathrm{mM} \mathrm{CaCl}_{2}$ before measuring the recycling pool.

To measure the fraction of transporter on the cell surface, surface fluorescence was quenched with $\mathrm{pH}$ 5.5 Tyrode's solution with HEPES replaced by MES. The transporter surface fraction was calculated by subtracting the average fluorescence intensity of five frames in $\mathrm{pH} 5.5$ Tyrode's solution from the average of five frames in standard $\mathrm{pH} 7.4$ Tyrode's solution (before stimulation) and divided by total fluorescence determined in the presence of $50 \mathrm{mM} \mathrm{NH}_{4} \mathrm{Cl}$. To estimate the change in fluorescence during stimulation to map the $\mathrm{N}$-terminal motifs (see Figs. $3,4)$, the average fluorescence at three time points $(27,30$, and $33 \mathrm{~s}$ ) in the middle of the stimulation period was used instead of the initial fluorescence at pH 7.4 in the above calculation. For these experiments only, four $400 \times 400$ pixel boxes were placed on each image field, avoiding cell bodies. Three $4 \times 4$ pixel boxes were placed in areas lacking cellular elements to measure background levels. The fluorescence of these regions was integrated and background subtracted.

Fluorescence measurements from 20 to 100 boutons per coverslip were averaged, and the means from 5 to 31 coverslips from at least two independent cultures were averaged, with the exception of $\Delta \mathrm{N}$-term \& $\Delta$ C-term VGLUT1 in Figure 3, which represents data from three coverslips from one culture. Data are presented as mean \pm SEM. The decay of poststimulus fluorescence, indicating endocytosis, and the fluorescence increase upon stimulation in the presence of bafilomycin were fit with single exponentials (GraphPad Prism). To determine statistical significance, one-way ANOVA with Tukey's post-test or an unpaired, twotailed $t$ test was performed, as appropriate (GraphPad Prism).

\section{Results}

\section{Mutation of the C-terminal dileucine-like motif fails to eliminate VGLUT1 endocytosis}

Since replacement of Phe-510 and Val-511 by alanine impairs the endocytosis of VGLUT1 but does not eliminate its synaptic targeting, response to stimulation, or recycling (Voglmaier et al., 2006) (Fig. 2), we also replaced these residues with glycine and compared the effect of these mutations in the VGLUT1-pHluorin reporter. Inserted into a luminal loop of the transporter, the fluorescence of ecliptic pHluorin (a modified form of GFP) is quenched at the low $\mathrm{pH}$ of synaptic vesicles (Miesenböck et al., 1998; Sankaranarayanan and Ryan, 2000). Exposure to the higher external $\mathrm{pH}$ upon exocytosis relieves this quenching, and reacidification after endocytosis again quenches the fluorescence. Changes in fluorescence intensity can thus monitor VGLUT1 exocytosis and endocytosis. We introduced the F510G/V511G (FV/GG) mutation into the VGLUT1-pHluorin fusion and transfected the resulting construct, along with wild-type (WT) and F510A/V511A (FV/AA), into rat hippocampal neurons. Upon stimulation of these cells at $30 \mathrm{~Hz}$ for $60 \mathrm{~s}$, we found that the FV/GG mutation impairs the compensatory endocytosis of VGLUT1 to a greater extent than the FV/AA mutation (Fig. 2A). After stimulation, endocytosis of the FV/GG mutant $\left(\tau_{\text {decay }}=\right.$ $89.4 \pm 10.0 \mathrm{~s})$ is significantly slower than WT $\left(\tau_{\text {decay }}=36.9 \pm\right.$ $4.3 \mathrm{~s} ; p<0.001)$ and FV/AA VGLUT1-pH $\left(\tau_{\text {decay }}=59.8 \pm 5.0 \mathrm{~s}\right.$; $p<0.01$ ) (Fig. 2B).

We also used quenching with Tyrode's solution buffered to pH 5.5 with MES to assess the cell surface levels of VGLUT1-pH at steady state, normalizing the results to total synaptic VGLUT1-pH revealed by alkalinization of the intracellular pool with $\mathrm{NH}_{4} \mathrm{Cl}$. The cell surface fraction of FV/GG VGLUT1-pH $(6.0 \pm 1.0 \%)$ is significantly higher than that of WT $(2.2 \pm 0.4 \%$; $p<0.01)$ or FV/AA transporters (3.2 $\pm 0.7 \%$; $p<0.05$ ) (Fig. $2 C$, solid bars). Three minutes after stimulation, the fluorescence of WT VGLUT1-pH returns to initial levels (WT, $2.3 \pm 0.9 \mathrm{~s}$ ), but a substantial fraction of the mutant transporters (FV/AA, $8.2 \pm$ $1.1 \%$; FV/GG, $18.8 \pm 1.4 \%$ ) remains on the cell surface, quenchable by Tyrode's solution at pH 5.5 (Fig. 2C, striped bars).

Since differences in exocytosis or total synaptic expression of the VGLUT1-pH constructs might have indirect effects on endocytosis, we also examined these properties. The vacuolar $\mathrm{H}^{+}$-ATPase inhibitor bafilomycin blocks synaptic vesicle reacidification when added to the imaging medium and thus eliminates the contribution of endocytosis to the fluorescence signal, revealing the total amount of transporter that undergoes exocytosis (Sankaranarayanan and Ryan, 2001). Stimulating in the presence of bafilomycin, we observe no significant difference between WT and the mutants in the fraction of the total internal pool of transporter that undergoes exocytosis in response to 30 Hz stimulation (WT, $69.6 \pm 4.1 \%$; FV/AA, $65.0 \pm 2.6 \%$; FV/GG, $65.8 \pm 2.2 \% ; p=0.55$ ) (Fig. $2 D$ ). The size of the internal trans- 

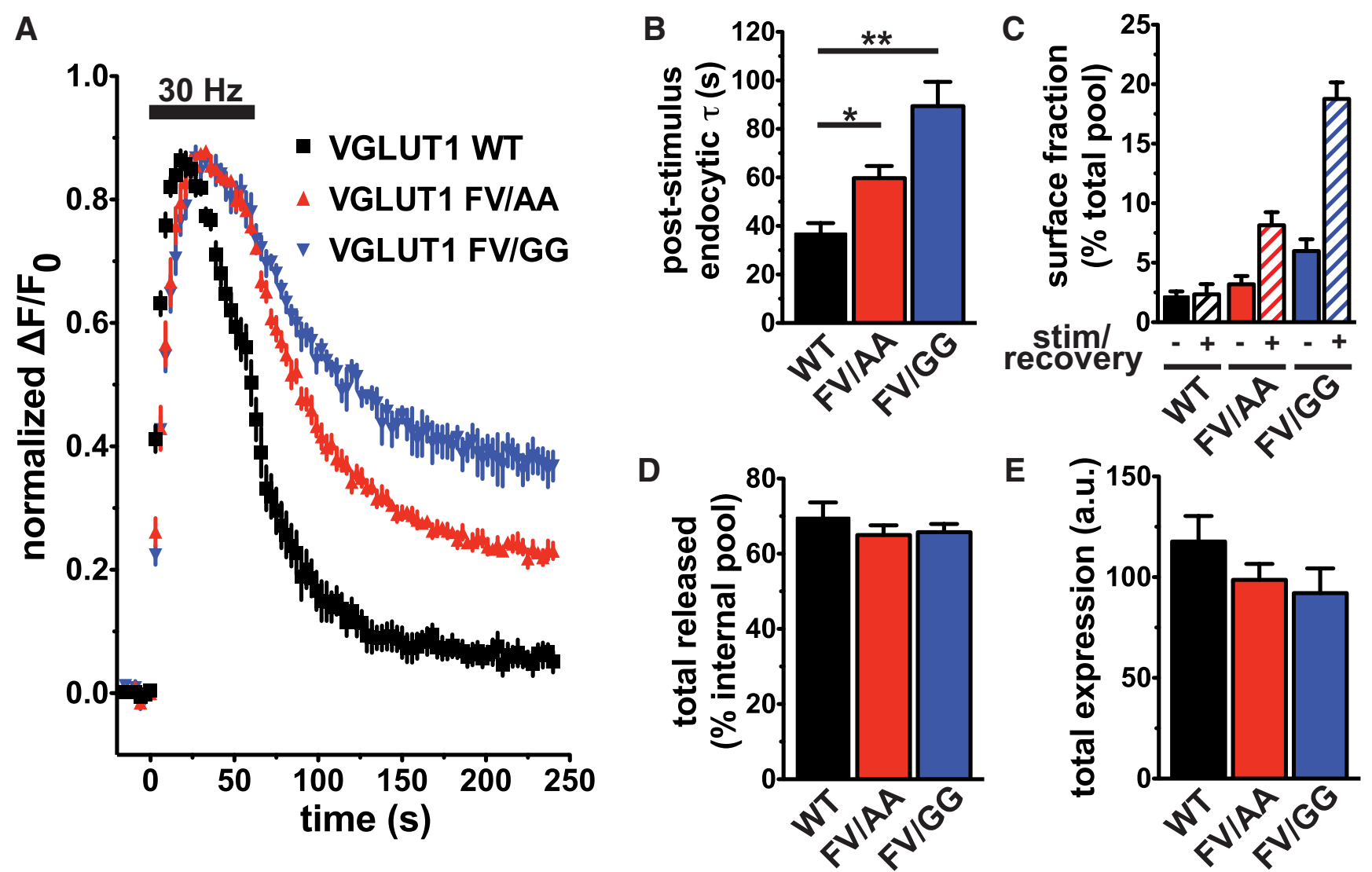

Figure 2. Mutation of the C-terminal dileucine-like motif slows, but does not eliminate VGLUT1 endocytosis. $A$, Time course of changes in fluorescence intensity $\left(\Delta F / F_{0}\right)$ in hippocampal neurons transfected with WT VGLUT1-pH (black), FV/AA VGLUT1-pH (red), or FV/GG VGLUT1-pH (blue). The traces, normalized to peak fluorescence, exhibit increases during stimulation at $30 \mathrm{~Hz}$ for 1 min (bar) and decreases after stimulation, consistent with exocytosis followed by endocytosis. Endocytosis of FV/GG VGLUT1-pH is less efficient than either WT or FV/AA. B, The mean poststimulus fluorescence decay, fit to a single exponential, is significantly faster for WT (36.9 $\pm 4.3 \mathrm{~s})$ than FV/AA (59.8 $\left.\pm 5.0 \mathrm{~s} ;{ }^{*} p<0.05\right)$ or FV/GG $\left(89.4 \pm 10.0\right.$ s; ${ }^{* *} p<0.001$, one-way ANOVA, Tukey's post-test). C, The fraction of VGLUT1-pH present on the cell surface at steady-state before stimulation (solid bars; stim/recovery - ) and 3 min after stimulation (striped bars; stim/recovery + ) was estimated by subtracting the fluorescence upon acid quenching with Tyrode's solution containing MES, pH 5.5, from the fluorescence at rest, normalized to total fluorescence upon alkalinization with $\mathrm{NH}_{4} \mathrm{Cl}$. The steady-state surface level of the FV/GG mutant $(6.0 \pm 1.0 \%)$ is significantly higher than both the FV/AA mutant $(3.2 \pm 0.7 \% ; p<0.05$, one-way ANOVA, Tukey's post-test) and WT $(2.2 \pm 0.4 \% ; p<0.01$, one-way ANOVA, Tukey's post-test). Fluorescence after recovery from stimulation of both FV/AA $(8.2 \pm 1.1 \%)$ and FV/GG $(18.8 \pm 1.4 \%)$ mutants are significantly higher than WT $(2.3 \pm 0.9 \%$; WT vs FV/AA, $p<0.01$; WT vs FV/GG, $p<0.001$; one-way ANOVA, Tukey's post-test). $\boldsymbol{D}$, The total amount of transporter released by stimulation at $30 \mathrm{~Hz}$ for 1 min was determined in the presence of bafilomycin. The fraction of the total internal pool released is similar between WT (69.6 $\pm 4.1 \%)$, FV/AA (65.0 $\pm 2.6 \%)$, and FV/GG (65.8 $\pm 2.2 \%)$ VGLUT1-pH ( $p=$ 0.55 , one-way ANOVA). The total internal pool was determined by subtraction of the initial fluorescence from the total fluorescence, as measured by application of $50 \mathrm{~mm} \mathrm{NH}_{4}\left(\mathrm{Cl}\right.$. $\boldsymbol{E}_{\text {, Total protein }}$ expression of WT (117.7 \pm 12.7 a.u.), FV/AA (98.7 \pm 7.9 a.u.), and FV/GG (92.1 \pm 12.2 a.u.) VGLUT1-pH at boutons are not significantly different ( $p=0.26$, one-way ANOVA). Data are the mean \pm SEM of at least 20 boutons per coverslip from $5-10$ coverslips from at least two independent cultures.

porter pool was determined by subtraction of the initial fluorescence (representing VGLUT1-pH molecules present on the cell surface before stimulation) from the total fluorescence, as measured with application of $\mathrm{NH}_{4} \mathrm{Cl}$ (Hua et al., 2011). In addition, alkalinization of the intracellular pool with $\mathrm{NH}_{4} \mathrm{Cl}$ shows no significant differences among the three proteins in total synaptic expression $(p=0.26)$ (Fig. 2E). These findings demonstrate that glycine mutations disrupt the endocytic role of the dileucine-like motif of VGLUT1 to a greater extent than alanine substitutions. However, a significant proportion of even the FV/GG mutant protein still localizes to synapses, responds to stimulation, and undergoes compensatory endocytosis, suggesting additional sorting signals.

\section{The $\mathrm{N}$ terminus of VGLUT1 contains additional} endocytic motifs

To identify additional endocytic motifs, we truncated either the cytosolic N or C terminus of VGLUT1 and examined the effects on behavior of the pHluorin fusions (Fig. 3). We first deleted the two polyproline domains downstream of the dileucine-like motif in the $\mathrm{C}$ terminus, including the second which interacts with endophilin (De Gois et al., 2006; Vinatier et al., 2006; Voglmaier et al., 2006; Weston et al., 2011). Replacement of Pro-530 with a stop codon in FV/GG VGLUT1-pH (FV/GG $\Delta$ PP) fails to eliminate the increase in fluorescence due to stimulation (from $12.4 \pm$ $2.6 \%$ at steady state to $43.1 \pm 3.1 \%$ of total pHluorin fluorescence during stimulation), indicating that the mutant transporter is still incorporated into synaptic vesicles (Fig. 3, top). Since the acidic residues (Glu-505 and Glu-506) upstream of the dileucine-like motif might still bind clathrin adaptor proteins (Owen et al., 2004), we next deleted nearly the entire VGLUT1-pH C terminus, replacing Ser-504 immediately upstream of the dileucine-like motif with a stop codon ( $\Delta \mathrm{C}$-term). However, $\Delta \mathrm{C}$-term produces no substantial change in steady-state surface level over FV/GG $\Delta \mathrm{PP}$ and also fails to eliminate the increase of fluorescence in response to stimulation (from $10.1 \pm 1.7 \%$ at steady state to $38.1 \pm 4.1 \%)$.

Deletion of the entire $\mathrm{N}$ terminus alone $(\Delta \mathrm{N}$-term) also fails to eliminate the stimulation-dependent increase in fluorescence, although the magnitude of the change appears to be smaller (from $6.8 \pm 0.8 \%$ before stimulation to $37.1 \pm 1.2 \%$ of total 


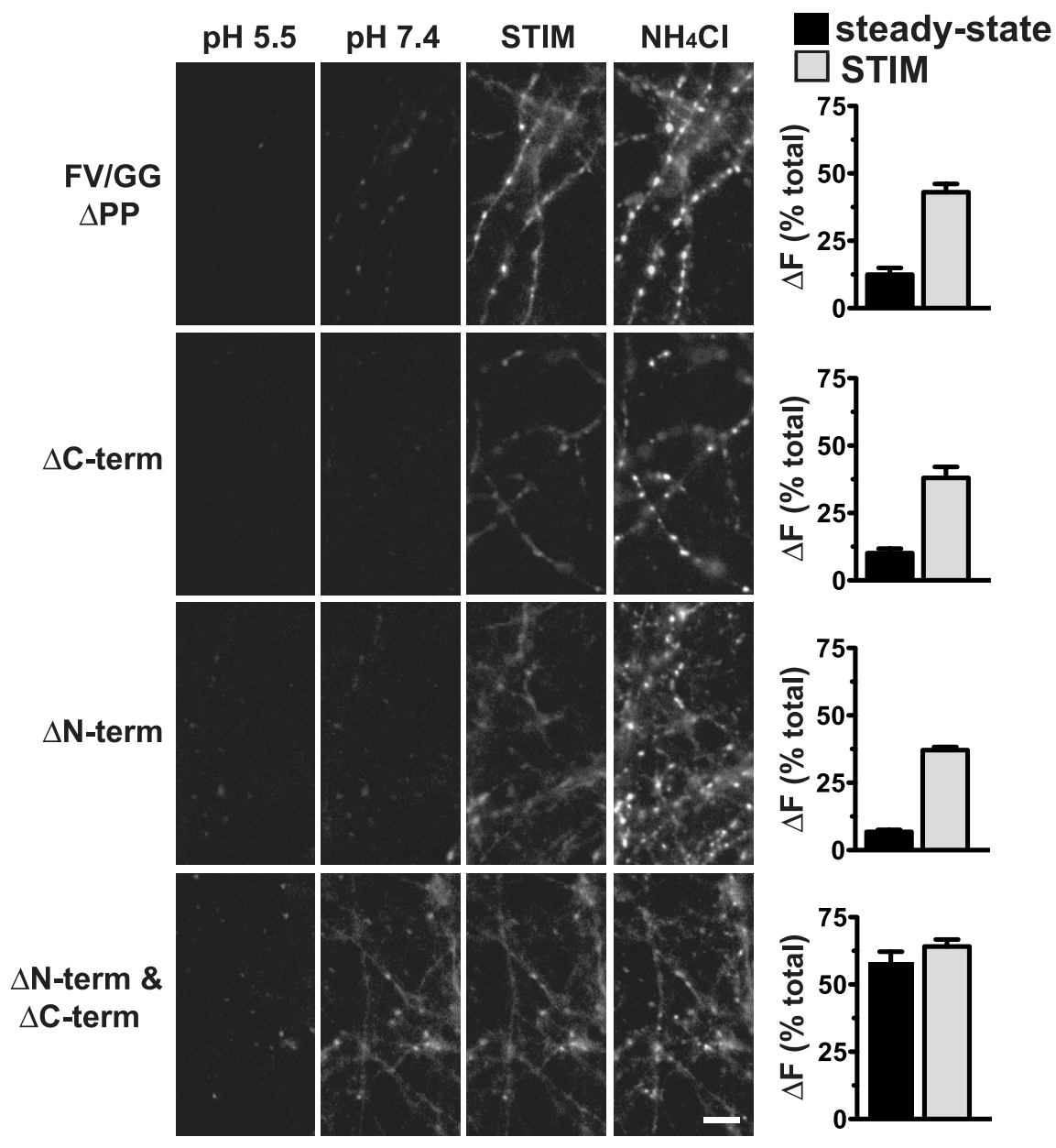

Figure 3. VGLUT1 requires either the N or C terminus to maintain an intracellular localization. Hippocampal neurons transfected with VGLUT1-pH truncation mutants were imaged in Tyrode's solution buffered with MES to pH 5.5 to quench surface fluorescence at rest, pH 7.4; after $30 \mathrm{~s}$ stimulation at $30 \mathrm{~Hz}$, near the peak of fluorescence (STIM); and upon alkalinization in $50 \mathrm{~mm} \mathrm{NH}_{4} \mathrm{Cl}$ to measure total fluorescence. Fluorescence at rest (steady state, black bars), normalized to unquenched fluorescence at pH 5.5 and total fluorescence $\left[\left(F_{\mathrm{pH} 7.4}-F_{\mathrm{pH} 5.5}\right) / F_{\text {total }}\right]$, increases during stimulation (STIM, gray bars) in neurons transfected with a VGLUT1-pH mutant that deletes the distal C terminus (FV/GG $\Delta$ PP; steady state, $12.4 \pm 2.6 \%$; STIM, $43.1 \pm 3.1 \%$ ), the full C terminus ( $\Delta$ C-term; steady state, $10.1 \pm 1.7 \%$; STIM, $38.1 \pm 4.1 \%$ ), or the full N terminus ( $\Delta \mathrm{N}$-term; steady state, $6.8 \pm 0.8 \%$; STIM, 37.1 $\pm 1.2 \%)$. In contrast, VGLUT1-pH lacking both the $\mathrm{N}$ and C termini ( $\Delta \mathrm{N}$-term \& $\Delta$ (-term) is highly expressed on the cell surface at steady state $(58.4 \pm 3.8 \%)$, and fluorescence is not significantly increased by stimulation $(64.1 \pm 2.5 \% ; p=0.28$, unpaired, two-tailed $t$ test). Data are the mean \pm SEM of 10-18 coverslips from at least four independent cultures with the exception of $\Delta \mathrm{N}$-term $\& \Delta$ (-term ( $n=3$ coverslips from one culture). Scale bar, $10 \mu \mathrm{m}$.

during stimulation) (Fig. 3). In contrast, VGLUT1-pH lacking both the $\mathrm{N}$ and $\mathrm{C}$ termini $(\Delta \mathrm{N}$-term \& $\Delta \mathrm{C}$-term) resides predominantly on the cell surface, is widely distributed along the processes rather than concentrated at synaptic sites, and does not respond to stimulation (fluorescence increases from $58.4 \pm 3.8 \%$ at steady state to only $64.1 \pm 2.5 \%$ during stimulation), indicating that the $\mathrm{N}$ terminus of VGLUT1 contains additional endocytic signals.

The heterotetrameric clathrin adaptor proteins (AP-1, AP-2, $\mathrm{AP}-3$ ) recognize both tyrosine-based motifs ( $\mathrm{Yxx} \phi$, where $\phi$ is a bulky, hydrophobic residue) and dileucine-like motifs, which can also contain acidic residues in the -4 position upstream ([DE] xxxL[ILM]) (Bonifacino and Traub, 2003). Inspection of the N-terminal sequence of VGLUT1 reveals two sequences that partially fit the consensus for a dileucine-like motif, ${ }_{6}$ EEFRKLA $_{12}$ and ${ }_{22} \mathrm{LL}_{23}$ (Fig. $4 A$ ). Since deletion of the $\mathrm{N}$ terminus alone has only a modest effect on the behavior of VGLUT1-pH, we introduced N-terminal glycine substitutions into $\Delta$ C-term VGLUT1-
$\mathrm{pH}$. In the first motif, mutation of Glu-6 and Glu-7 to glycine in $\Delta$ C-term VGLUT1-pH (EE/GG $\Delta$ C-term) does not replicate the effect of the $\Delta \mathrm{N}$-term \& $\Delta \mathrm{C}$ term double truncation (Fig. $4 B$ ). A substantial fraction of the EE/GG $\Delta$ C-term mutant is internalized at steady state (steady-state surface level, $16.6 \pm 2.7 \%$ total) and a large increase in fluorescence is observed with stimulation, indicating exocytosis (to $55.5 \pm 1.6 \%$ total). However, replacement of Leu-11 and Ala-12 with glycine in addition to the EE/GG mutation and $\Delta$ C-term (EELA/GGGG $\Delta$ Cterm) greatly increases steady-state surface expression $(47.0 \pm 1.8 \%$ total $)$ (Fig. 4B). Stimulation still produces a significant, albeit small, increase in fluorescence (to $65.4 \pm 1.9 \%$ total; $p<0.0001)$

In the second $\mathrm{N}$-terminal motif, replacement of Leu-22 and Leu-23 by glycine in $\Delta$ C-term VGLUT1-pH (LL/GG $\Delta \mathrm{C}$-term) increases steady-state surface expression even further (to $68.5 \pm 2.5 \%$ total), with no significant fluorescence increase in response to stimulation (73.0 \pm $1.8 \% ; p=0.16$ ) (Fig. $4 B$ ). Mutation of both ${ }_{11} \mathrm{LA}_{12}$ and ${ }_{22} \mathrm{LL}_{23}$ motifs in the C-terminal truncation (LALL/GGGG $\Delta$ C-term) increases surface expression to similar levels $(63.5 \pm 3.0 \%)$ with no significant fluorescence increase in response to stimulation $(63.1 \pm 3.3 \% ; p=0.92)$. Thus, mutation of either ${ }_{11} \mathrm{LA}_{12}$ or ${ }_{22} \mathrm{LL}_{23}$ to glycine in the context of the C-terminal deletion can mimic the synaptic trafficking defect of the $\Delta \mathrm{N}$-term \& $\Delta \mathrm{C}$-term double truncation mutant, indicating that two additional dileucine-like signals are present in the $\mathrm{N}$ terminus of VGLUT1.

Interestingly, all three dileucine-like motifs in VGLUT1 are atypical. The most common definition for a dileucine motif is [DE] $\mathrm{xxxL}[\mathrm{ILM}]$ (Bonifacino and Traub, 2003). The N-terminal ${ }_{6}$ EEFRKLA $_{12}$ and C-terminal ${ }_{505} \mathrm{EEKCGFV}_{511}$ have an acidic residue in the -4 position, but contain atypical residues (A, F, and V), instead of the standard hydrophobic residues ( $\mathrm{L}, \mathrm{I}$, or $\mathrm{M})$. This suggests that a wider range of amino acid residues may substitute for leucine. These motifs do fit the requirement that the acidic residues are $\mathrm{N}$-terminal to the hydrophobic residues, and are at least six residues from the transmembrane helix (Geisler et al., 1998). The second N-terminal motif $\left({ }_{22} \mathrm{LL}_{23}\right)$ is atypical since it consists of only two leucines without upstream acidic residues. In addition, the $\mathrm{N}$-terminal location positions both motifs in the opposite orientation relative to the membrane from more typical C-terminal motifs. However, several other proteins contain N-terminal dileucine-like motifs including VAMP4 and the CLN7 lysosomal glycoprotein (Peden et al., 2001; Steenhuis et al., 2010). In addition, at least one other protein, mucolipin, contains both $\mathrm{N}$ - and C-terminal dileucine-like motifs that mediate distinct trafficking steps (Vergarajauregui and Puertollano, 2006). 
To determine whether the N-terminal motifs can mediate endocytosis, we measured the internalization of chimeras containing VGLUT1 N-terminal (NTD) and C-terminal domains (CTD) fused to the short cytoplasmic tail of the interleukin 2 receptor $\alpha$-subunit, commonly known as Tac, a resident plasma membrane protein (Bonifacino et al., 1990; Tan et al., 1998; Hawryluk et al., 2006; Voglmaier et al., 2006). Transfected HeLa cells expressing the Tac chimeras were incubated with an antibody to the ectodomain of Tac at $4^{\circ} \mathrm{C}$ (to inhibit endocytosis), allowed to internalize at $37^{\circ} \mathrm{C}$ for $1 \mathrm{~h}$, stained without permeabilization using a FITC-conjugated secondary antibody, and then permeabilized and stained with a second, Cy3conjugated secondary antibody (Fig. 5A). Thus, surface Tac is labeled with FITC, and total (surface and internalized) Tac is labeled with Cy3. We compared surface and total immunostaining of Tac chimeras of the VGLUT1 NTD (Fig. 5B) and the VGLUT1 CTD, and related mutants. Tac chimeras of the vesicular monoamine transporter VMAT2 C terminus, containing a previously characterized dileucinelike motif, served as a control (Tan et al., 1998) (Fig. 5C,D). To quantify the extent of internalization of the different Tac chimeras, we calculated the correlation coefficient $\left(r_{\text {avg }}\right)$ of colocalization of surface (green) and total (red) staining (Fig. 5C). A larger correlation coefficient corresponds to greater surface localization, indicating less internalization.

Unmodified Tac is labeled by both FITC- and Cy3-conjugated antibodies, demonstrating a lack of internalization from the plasma membrane $\left(r_{\text {avg }}=\right.$ $0.87 \pm 0.01)$ (Fig. $5 C, D$ ). In contrast, fusion of the VMAT2 $\mathrm{C}$ terminus to Tac results in the appearance of intracellular red puncta, representing internalized Tac chimeras $\left(r_{\text {avg }}=0.50 \pm 0.02 ; p<\right.$ 0.001 relative to Tac). Mutation of the well-characterized dileucine-like motif (VMAT2 IL/AA) impairs endocytosis of VMAT2, as described previously $\left(r_{\mathrm{avg}}=\right.$ $0.81 \pm 0.01, p<0.05$ relative to Tac; $p<$ 0.001 relative to VMAT2) (Tan et al., 1998). Similar to VMAT2, addition of the VGLUT1 CTD facilitates antibody internalization $\left(\mathrm{CTD}, r_{\mathrm{avg}}=0.61 \pm 0.02 ; p<0.001\right.$ relative to $\mathrm{Tac}$ ), and mutation of the dileucine-like motif (VGLUT1 CTD FV/GG) also impairs internalization (CTD FV/GG, $r_{\text {avg }}=$ $0.87 \pm 0.01 ; p>0.05$ relative to Tac, $p<0.001$ relative to CTD). The N-terminal domain (VGLUT1 NTD) confers a significant degree of internalization compared to Tac $\left(r_{\text {avg }}=\right.$ $0.78 \pm 0.01 ; p<0.001$ relative to Tac), but a substantial fraction of surface labeled protein persists after $1 \mathrm{~h}$. Confirming the role of the $\mathrm{N}$-terminal dileucine-like motifs in endo-

B

\section{LALL/GGGG $\Delta \mathbf{C}$-term}

\section{A}

$$
\text { pH } 5.5
$$
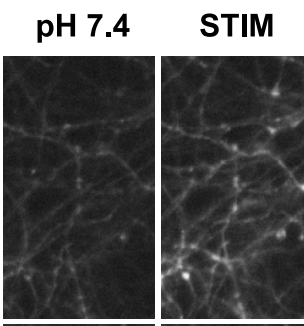

$\mathrm{NH}_{4} \mathrm{Cl}$
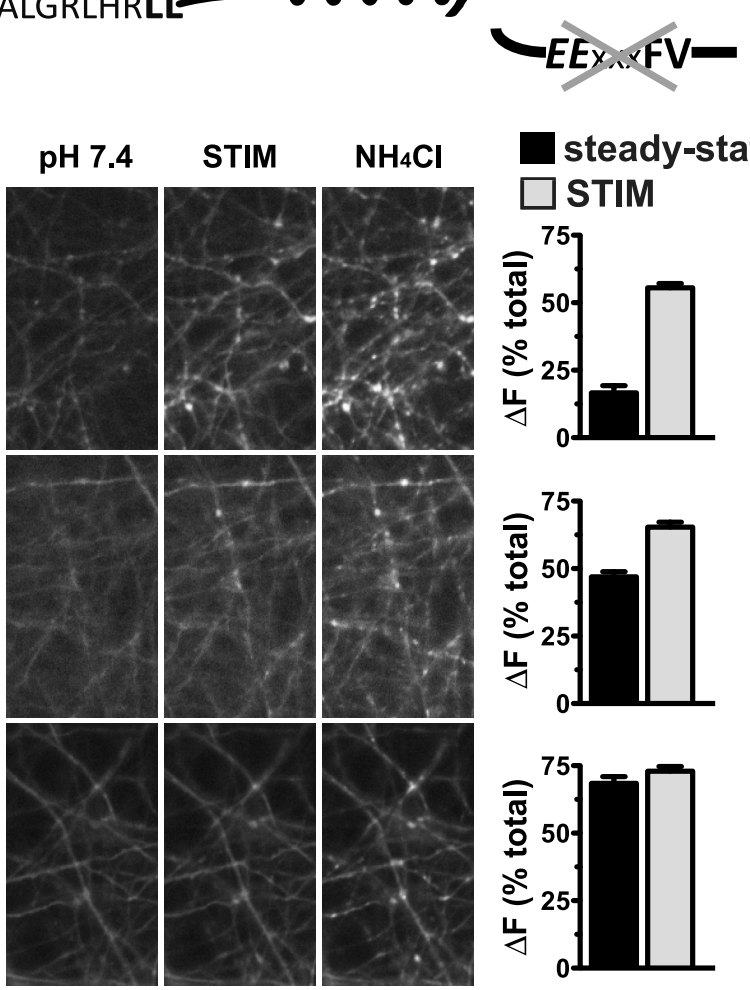

Figure 4. The N terminus of VGLUT1 contains two dileucine-like motifs. A, Diagram of VGLUT1-pH highlighting the N and C termini. Residues, in bold, in two N-terminal dileucine-like motifs were mutated to glycine in the context of the full C-terminal deletion. $\boldsymbol{B}$, Point mutations were made in the $\mathrm{N}$ terminus of $\Delta \mathrm{C}$-term VGLUT1-pH and constructs transfected into hippocampal neurons stimulated at $30 \mathrm{~Hz}$ for $1 \mathrm{~min}$. Shown are representative fluorescence images of cells in Tyrode's solution buffered with MES to pH 5.5 to quench surface fluorescence; at rest, pH 7.4; after 30 s stimulation, near the peak of fluorescence (STIM); and upon alkalinization in $50 \mathrm{~mm} \mathrm{NH}_{4} \mathrm{Cl}$ to measure total fluorescence. Fluorescence levels, normalized to unquenched fluorescence at pH 5.5 and total fluorescence $\left[\left(F_{\mathrm{pH} 7.4}-F_{\mathrm{pH} 5.5}\right) / F_{\text {total }}\right.$, before stimulation (black bars) were compared to fluorescence levels after $30 \mathrm{~s} \mathrm{of}$ stimulation (gray bars). Mutation of the acidic residues in the first dileucine-like motif to glycine (EE/GG $\Delta C$-term) does not eliminate the increase in fluorescence in response to stimulation (steady state, $16.6 \pm 2.7 \%$; STIM, $55.5 \pm 1.6 \%$ ). Additional mutation of ${ }_{11} \mathrm{LA}_{12}$ (EELA/GG $\Delta \mathrm{C}$-term) increases steady-state fluorescence levels (47.0 $\pm 1.8 \%$ ), which increase further upon stimulation (STIM, 65.4 $\pm 1.9 \%$ ). No fluorescence increase upon stimulation is observed with mutation of ${ }_{22} \mathrm{LL}_{23}\left(\mathrm{LL} / \mathrm{GG} \Delta \mathrm{C}\right.$-term; steady state, $68.5 \pm 2.5 \%$; $\mathrm{STIM}, 73.0 \pm 1.8 \%$ ) or with mutation of both ${ }_{22} \mathrm{LL}_{23}$ and ${ }_{11} \mathrm{LA}_{12}$ (LALL/GGGG $\Delta$ C-term; steady state, $63.5 \pm 3.0 \% ;$ STIM, 63.1 $\pm 3.3 \%$ ). Data are the mean \pm SEM of $8-10$ coverslips from two independent cultures. Scale bar, $10 \mu \mathrm{m}$.

cytosis, mutation of Glu-6, Glu-7, Leu-11, Ala-12, Leu-22, and Leu-23 in the N-terminal chimera (VGLUT1 NTD NT3) largely eliminates internalization $\left(r_{\mathrm{avg}}=0.88 \pm 0.01 ; p>0.05\right.$ relative to Tac, $p<0.001$ relative to NTD) (Fig. $5 C, D$ ). Tac chimeras containing the individual $\mathrm{N}$-terminal domain mutants (VGLUT1 NTD NT1 or NT2) do not significantly differ from Tac in the extent of internalization (NTD NT1, $r_{\text {avg }}=$ $0.83 \pm 0.01 ;$ NTD NT2, $r_{\text {avg }}=0.86 \pm 0.01$; both $p>0.05$ relative to $\mathrm{Tac}$ ). 
A

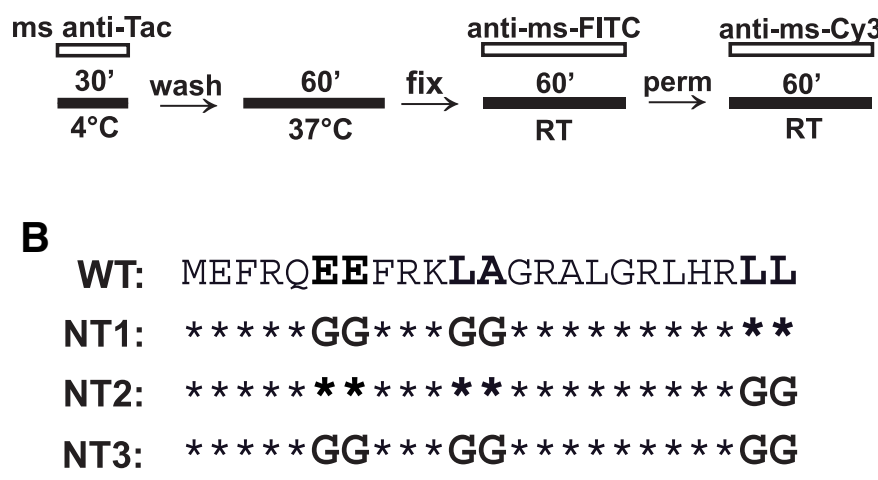

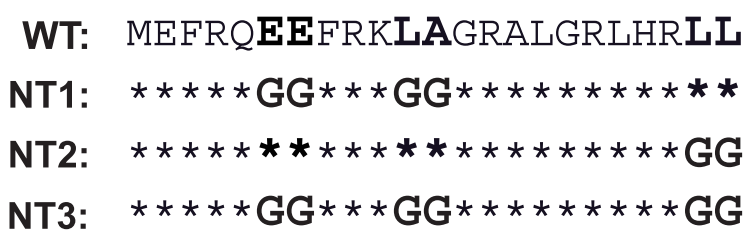

C

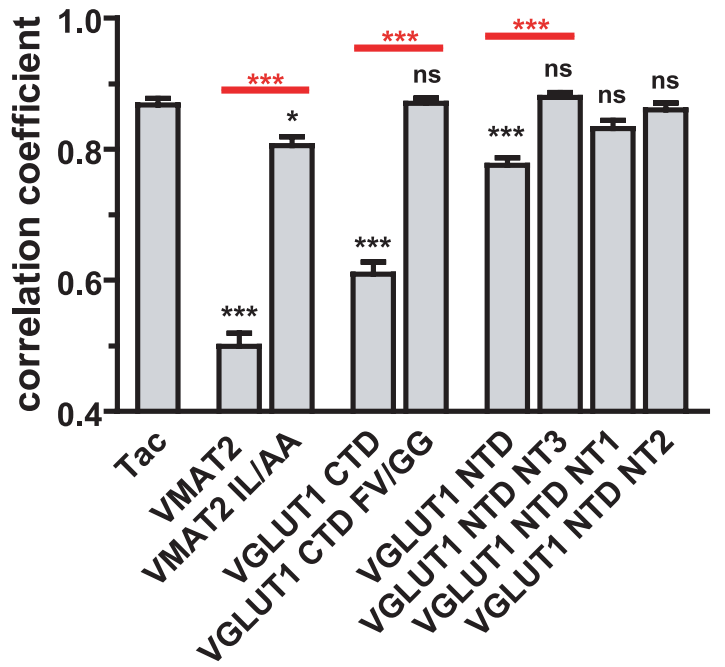

D
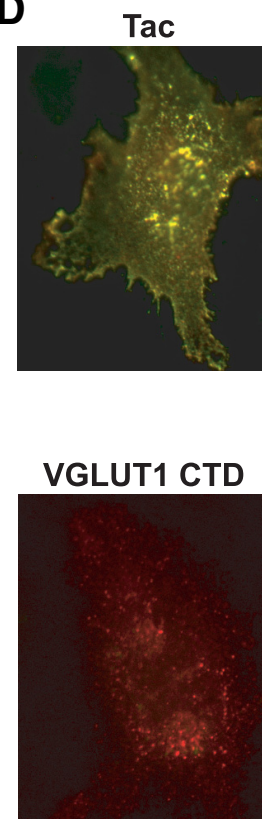

VGLUT1 NTD NT1

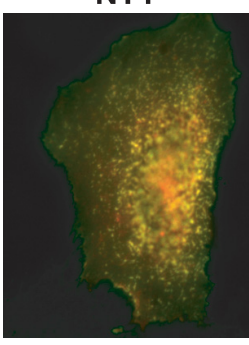

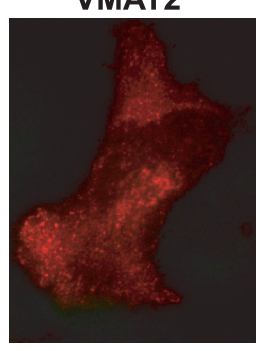

VGLUT1 CTD

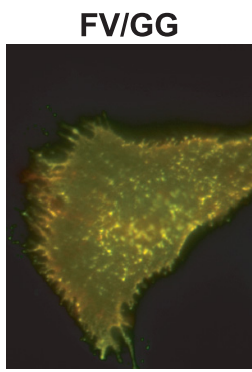

VMAT2 ILIAA
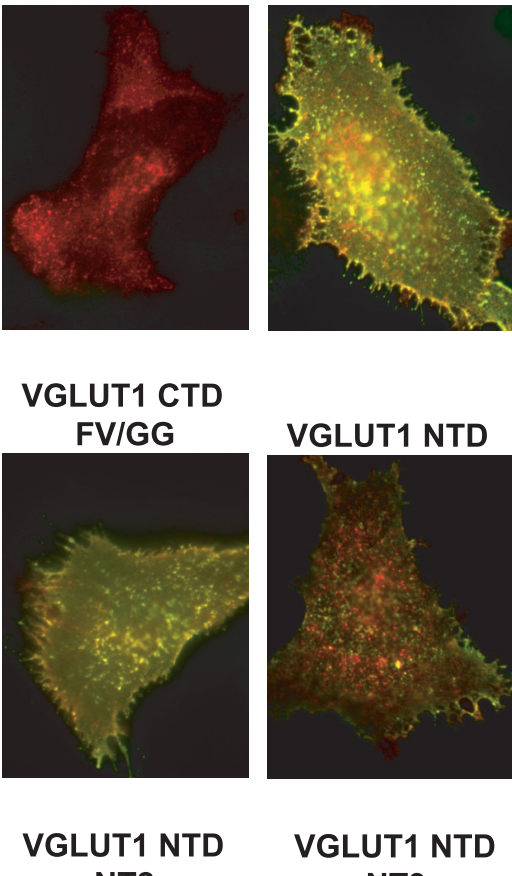
NT2

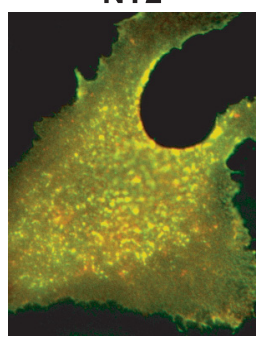

VGLUT1 NTD NT3

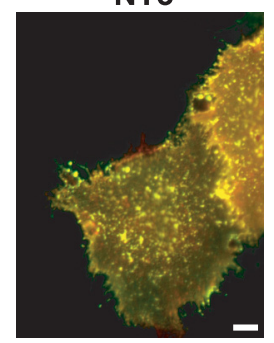

Figure 5. The N-terminal dileucine-like motifs are sufficient to facilitate endocytosis. $\boldsymbol{A}$, Diagram of staining procedure. HeLa cells transfected with the indicated Tac chimeras were incubated with mouse (ms) anti-Tac antibody for $30 \mathrm{~min}$ at $4^{\circ} \mathrm{C}$. After removal of unbound antibody, cells were incubated at $37^{\circ} \mathrm{C}$ for $1 \mathrm{~h}$ to allow internalization. Cells were then fixed and incubated for $1 \mathrm{~h}$ with a FITC-conjugated secondary antibody to visualize surface Tac. To measure total Tac, cells were then permeabilized and stained with a Cy3-conjugated secondary antibody. $\boldsymbol{B}$, Diagram of residues mutated to disrupt the N-terminal dileucine-like motifs of VGLUT1. To disrupt the first motif, Glu-6, Glu-7, Leu-11, and Ala-12 were replaced with glycine (NT1). Leu-22 and Leu-23 were replaced to eliminate the second motif (NT2). Mutation of all six residues disrupts both N-terminal motifs (NT3). C, To quantify the extent of internalization of the various Tac chimeras, colocalization of surface (green) and total (red) staining was measured and is represented as an average correlation coefficient $\left(r_{\text {avg }}\right)$ for $>60$ cells per construct from two independent transfections. Compared to Tac alone, the $C$ termini of both VMAT2 $\left(r_{\text {avg }}=0.50 \pm 0.02 ; p<0.001\right)$ and VGLUT1 $\left(r_{\text {avg }}=0.61 \pm 0.02 ; p<0.001\right)$ are capable of conferring significant internalization onto Tac. In both cases, internalization is largely eliminated by mutation of the respective dileucine-like motifs [VMAT2 IL/AA, $r_{\text {avg }}=0.81 \pm 0.01, p<0.05$ relative to Tac (black), $p<0.001$ relative to WT VMAT2 (red); VGLUT1 CTD FV/GG, $r_{\text {avg }}=0.87 \pm 0.01, p>0.05$ relative to Tac (black), $p<0.001$ relative to VGLUT1 CTD (red)]. The N terminus of VGLUT1 is also sufficient to confer internalization on Tac but to a lesser degree than the VGLUT1 C terminus [VGLUT1 NTD, $r_{\text {avg }}=0.78 \pm 0.01 ; p<0.001$ relative to Tac (black)]. Internalization conferred by the VGLUT1 NTD is eliminated by mutation of both dileucine-like motifs [VGLUT1 NT3, $r_{\text {avg }}=0.88 \pm 0.01 ; p>0.05$ relative to Tac (black), $p<0.001$ relative to VGLUT1 NTD (red)]. Mutation of either motif individually also appears to disrupt internalization (NT1, $r_{\text {avg }}=0.83 \pm 0.01 ; \mathrm{NT2}, r_{\text {avg }}=0.86 \pm 0.01$, both $p>0.05$ relative to Tac). Statistical differences relative to Tac are represented above each bar as black symbols, and differences between WT and corresponding dileucine mutant chimeras are indicated by red symbols and bars. ${ }^{*} p<0.05 ;{ }^{* * *} p<0.001$. ns, Not significant. Data are shown as mean \pm SEM. All $p$ values were calculated using one-way ANOVA and Tukey's post-test. $\boldsymbol{D}$, Representative images of cells quantified in $\boldsymbol{C}$. Scale bar, $10 \mu \mathrm{m}$.

N-terminal dileucine-like motifs influence cell surface expression of VGLUT1-pH independent of effects on compensatory endocytosis

To assess an independent role for the N-terminal dileucine-like motifs in VGLUT1 trafficking, we studied the effect of $\mathrm{N}$-terminal mutations in the context of the intact cytoplasmic C terminus. Simultaneous replacement of Glu-6, Glu-7, Leu-11, Ala-12, Leu-22, and Leu-23 with glycine (NT3) increases the surface fraction of VGLUT1-pH $(6.3 \pm 0.2 \%)$ relative to WT $(2.1 \pm$ $0.3 \% ; p<0.001$ ) (Fig. $6 A$ ). However, mutation of either the first (NT1) or the second (NT2) dileucine-like motif alone does not increase steady-state cell surface fraction (NT1, $2.5 \pm 0.7 \%$; NT2, $2.5 \pm 0.5 \%)$, and the differences in surface fraction do not reflect differences in protein expression (Fig. 6B).
We then examined the exocytosis of both the $\mathrm{N}$ - and C-terminal mutants, determining the size of the RRP and RP as a fraction of the total internal pool of VGLUT1-pH. The internal pool was measured by subtraction of the initial fluorescence from the total fluorescence as revealed in $\mathrm{NH}_{4} \mathrm{Cl}$ (Hua et al., 2011). To determine the size of the RRP and RP, we evoked release and trapped VGLUT1-pH in the alkaline, fluorescent state by blocking acidification with bafilomycin as described above (Sankaranarayanan and Ryan, 2001). Eliciting release of vesicles from the RRP with $100 \mathrm{~Hz}$ stimulation for $0.2 \mathrm{~s}$, we observed no significant differences in the fraction of WT, NT3, or FV/GG VGLUT1-pH that undergoes exocytosis (WT, $5.0 \pm 0.8 \%$; NT3, $3.6 \pm 0.3 \%$; FV/GG, $4.8 \pm 0.8 \%$; $p=0.24$ ) (Fig. 6C). Similarly, release of the RP with $10 \mathrm{~Hz}$ 

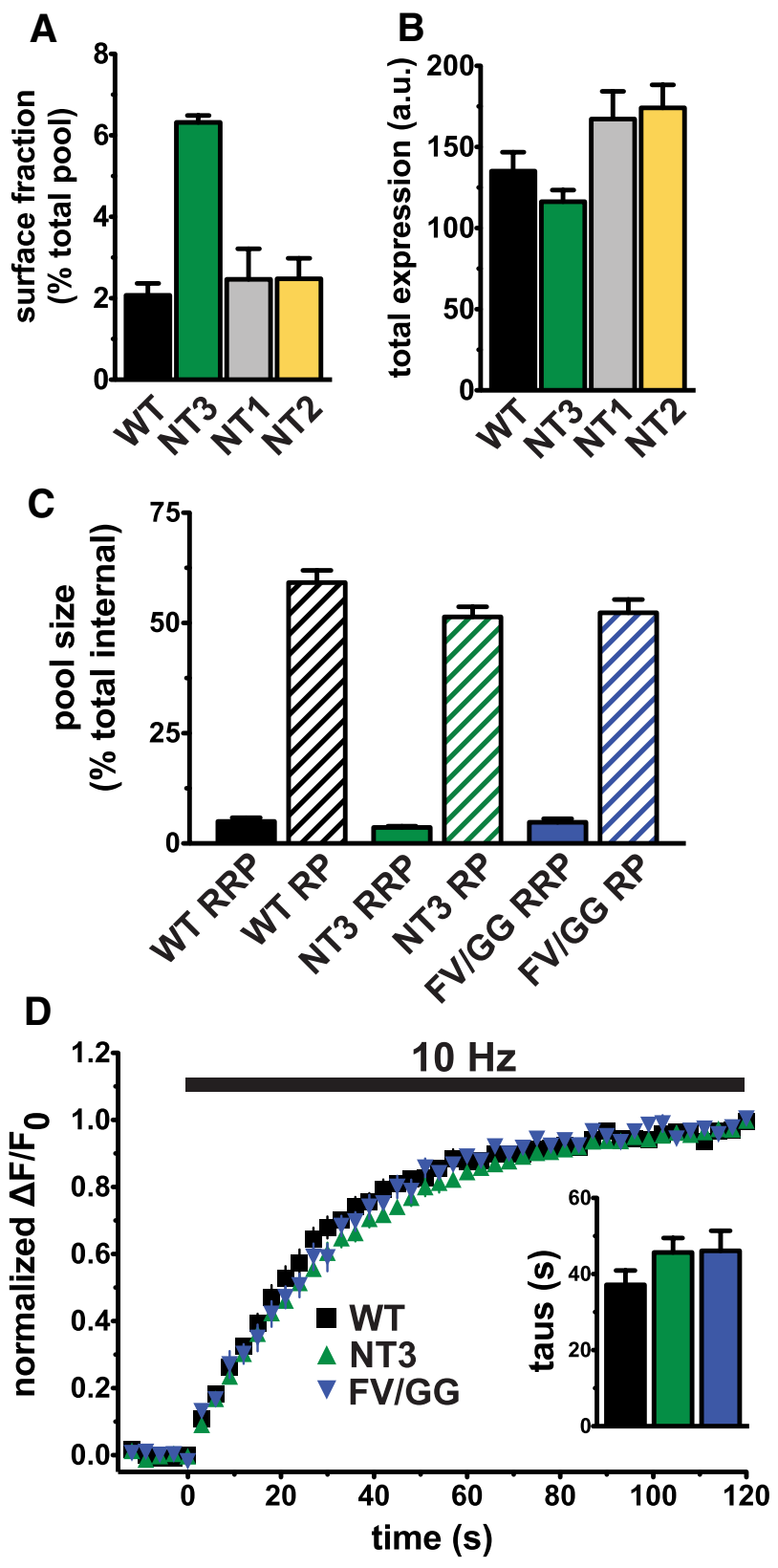

Figure 6. Mutation of $\mathrm{N}$-terminal dileucine-like motifs increases cell surface expression. $\boldsymbol{A}$, The surface fraction of NT3 $(6.3 \pm 0.2 \%)$ is significantly higher than either WT $(2.1 \pm 0.3 \% ; p<0.001)$ or either individual $\mathrm{N}$-terminal mutant, NT1 $(2.5 \pm 0.7 \% ; p<0.01)$ or NT2 $(2.5 \pm 0.5 \% ; p<0.01$, one-way ANOVA, Tukey's post-test). $\boldsymbol{B}$, Total expression level of NT1 (167.2 \pm 17.1 a.u.; $p>0.05$ ), NT2 (174.2 \pm 14.2 a.u.; $p>0.05$ ), and NT3 (116.3 \pm 7.2 a.u; $p>0.05$ ) are not different than WT (135.1 \pm 11.7 a.u.; one-way ANOVA, Tukey's post-test). C, The size of the readily releasable pool, as measured with a $100 \mathrm{~Hz}, 200$ ms stimulus, is not significantly altered by mutation of either the $\mathrm{N}$ - or C-terminal dileucine-like motifs (WT, $5.0 \pm 0.8 \% ; \mathrm{NT} 3,3.6 \pm 0.3 \% ; \mathrm{FV} / \mathrm{GG}, 4.8 \pm 0.8 \% ; p=0.24$, one-way ANOVA). Recycling pool size, as measured by a $10 \mathrm{~Hz}, 90$ s stimulus, is also not significantly different for WT (59.2 $\pm 2.7 \%)$, NT3 (51.4 $\pm 2.3 \%)$, and FV/GG (52.3 $\pm 3.0 \%$; $p=0.11$, one-way ANOVA). Pool sizes are expressed as a fraction of the total internal pool, determined by subtraction of the initial fluorescence from total fluorescence. $\boldsymbol{D}$, The rate of exocytosis in response to a $10 \mathrm{~Hz}, 120 \mathrm{~s}$ stimulus is not significantly different between WT (black; $\tau=37.2 \pm 3.7 \mathrm{~s}$ ), NT3 (green; $\tau=$ $45.7 \pm 3.8 \mathrm{~s}$ ), and FV/GG (blue; $\tau=46.1 \pm 5.3 \mathrm{~s} ; p=0.28$, one-way ANOVA). Data are the mean \pm SEM of 8-31 coverslips from at least three independent cultures with at least 20 synapses averaged per coverslip.

stimulation for $90 \mathrm{~s}$ shows no significant differences in RP size (WT, $59.2 \pm 2.7 \%$; NT3, $51.4 \pm 2.3 \%$; FV/GG, $52.3 \pm 3.0 \%$; $p=0.11$ ) (Fig. 6C). The rate of exocytosis in response to $10 \mathrm{~Hz}$ stimulation also shows no significant differences among the three genotypes (WT, $\tau=37.2 \pm 3.7 \mathrm{~s}$; NT3, $\tau=45.7 \pm 3.8 \mathrm{~s}$; FV/GG, $\tau=46.1 \pm 5.3 \mathrm{~s} ; p=0.28$ ) (Fig. $6 D$ ).

Since the increased steady-state surface expression of the $\mathrm{N}$-terminal mutant might reflect a defect in compensatory endocytosis, we examined the endocytosis of VGLUT1 after stimulation at a range of frequencies. Disruption of the N-terminal motifs did not significantly alter the recycling of VGLUT1-pH with stimulation at $5 \mathrm{~Hz}$ for $5 \mathrm{~min}$ (Fig. 7A), $10 \mathrm{~Hz}$ for $1 \mathrm{~min}(B)$, $30 \mathrm{~Hz}$ for $1 \mathrm{~min}(C)$, or $80 \mathrm{~Hz}$ for $1 \mathrm{~min}(D)$, in contrast to the obvious impairment caused by C-terminal FV/GG and FV/AA mutations (Fig. 2) (Voglmaier et al., 2006).

Although we did not observe a defect in endocytic rate with mutation of the N-terminal motifs alone, the N-terminal motifs presumably mediate the residual endocytosis observed with the FV/GG mutation. A substantial portion of the FV/GG VGLUT1 mutant that is exocytosed upon stimulation is reinternalized (Fig. $2 A, C, D)$. Consistent with this, the low level of steady-state surface expression of this mutant $(\sim 6 \%)$ (Fig. $2 C$ ) supports internalization by some other mechanism, and the $\mathrm{C}$ terminus cannot be responsible because $\Delta$ C-term VGLUT1-pH also remains largely intracellular and responsive to stimulation (Fig. 3). In addition, the endocytosis of $\Delta \mathrm{C}$-term VGLUT1-pH is not slower than the FV/GG mutant, suggesting that the additional internalization signal does not reside in the $\mathrm{C}$ terminus (data not shown). Whereas the $\mathrm{N}$-terminal domains may thus partially compensate for loss of the C-terminal dileucine-like motif, the C-terminal dileucine-like motif more fully compensates for loss of the $\mathrm{N}$-terminal motifs. Thus, the $\mathrm{N}$ - and C-terminal motifs may promote VGLUT1-pH recycling by different mechanisms.

\section{$\mathrm{N}$ - and C-terminal dileucine-like motifs are recognized by different adaptor proteins}

To characterize the endocytic mechanisms recruited by the $\mathrm{N}$ and C-terminal motifs of VGLUT1, we used previously published shRNA sequences to reduce the levels of the clathrin adaptors AP-1 and AP-2 in hippocampal neurons (Dugast et al., 2005; Kim and Ryan, 2009; Cheung and Cousin, 2012). Neurons were transfected at the time of plating with WT, FV/GG, or NT3 VGLUT1-pH and infected at DIV7-DIV8 with lentiviruses expressing constructs containing either AP- $2 \mu$-specific or two different AP- $1 \gamma$-specific shRNA hairpins, along with BFP as a reporter to assess infection efficiency. Lentivirus expressing the empty vector was used as a control. As the only isoform of their subunit in the two adaptors, loss of AP- $1 \gamma$ and AP- $2 \mu$ destabilizes the other subunits and hence inactivates the entire complex (Kantheti et al., 1998; Meyer et al., 2000; Motley et al., 2003). Western analysis of infected hippocampal neurons indeed confirms a specific reduction in the levels of AP-1, but not AP-2 or AP-3, after infection with virus expressing either of the two AP1-targeted hairpins (Fig. 8A). AP-1 knockdown was also confirmed by immunostaining randomly selected coverslips after live cell imaging (data not shown). The AP-2 shRNA construct (Kim and Ryan, 2009) results in a similar reduction in AP-2, but not of AP-1 or AP-3 (data not shown).

At DIV14-DIV17, the neurons were stimulated at $30 \mathrm{~Hz}$ for 1 min and the fluorescence monitored. Depletion of AP-2 slows the rate of poststimulus endocytosis of WT VGLUT1-pH $\left(\tau_{\text {decay }}=\right.$ $67.1 \pm 8.5 \mathrm{~s})$ relative to the vector control $\left(\tau_{\text {decay }}=39.5 \pm 4.2 \mathrm{~s}\right.$; $p<0.05$ ) (Fig. $8 B$ ), consistent with previous work using the same shRNA sequence (Kim and Ryan, 2009). However, NT3 VGLUT1-pH shows even more sensitivity to AP-2 knockdown with $\tau_{\text {decay }}=138.2 \pm 19.4 \mathrm{~s}$ relative to $\tau_{\text {decay }}=51.7 \pm 5.2 \mathrm{~s}$ for the control $(p<0.001)$ (Fig. $8 C$ ). Since the endocytosis of 
A
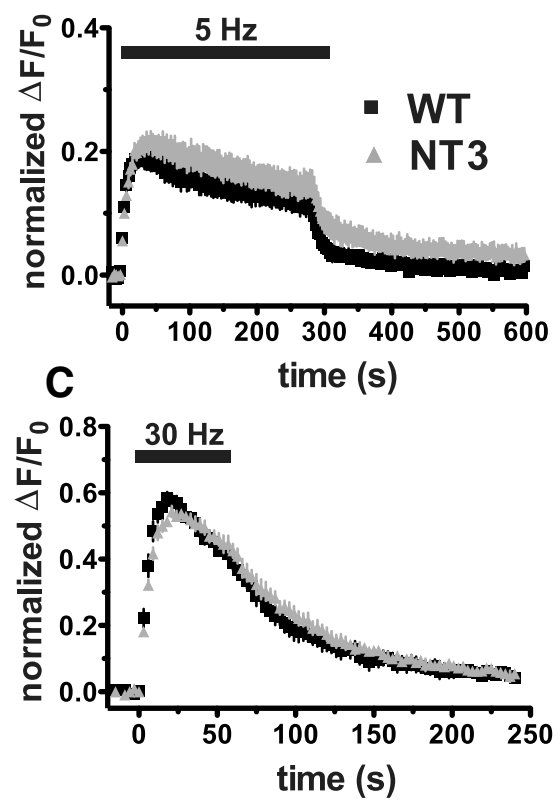

B
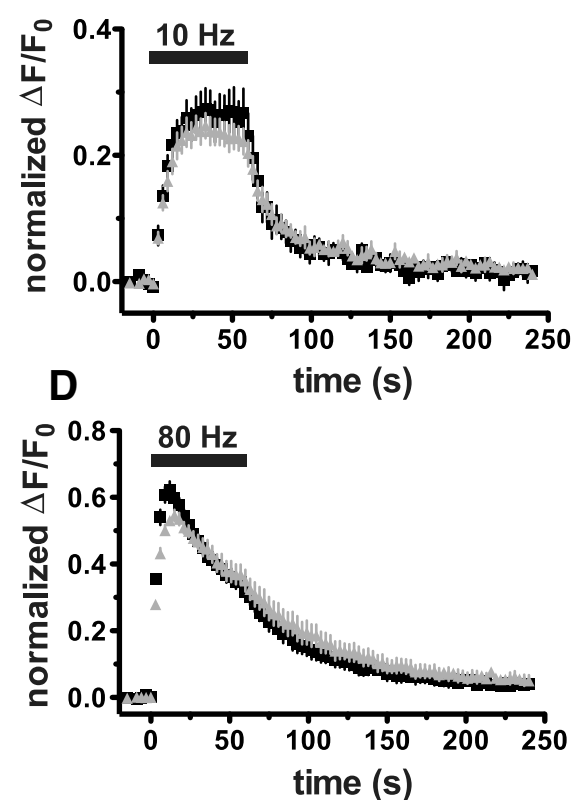

Figure 7. Mutation of the N-terminal dileucine-like motifs does not significantly impair endocytosis. $\boldsymbol{A}-\boldsymbol{D}$, Time course of fluorescence changes in neurons transfected with WT VGLUT1-pH (black) or NT3 VGLUT1-pH (gray) with $5 \mathrm{~Hz}$ for $5 \mathrm{~min}(\boldsymbol{A}), 10 \mathrm{~Hz}$ for $1 \mathrm{~min}(\boldsymbol{B}), 30 \mathrm{~Hz}$ for $1 \mathrm{~min}(\boldsymbol{C})$, or $80 \mathrm{~Hz}$ for $1 \mathrm{~min}(\boldsymbol{D})$ stimulation (black bars). Each trace was normalized to the size of the total pool of VGLUT1-pH as determined by application of modified Tyrode's solution containing $50 \mathrm{~mm} \mathrm{NH}_{4} \mathrm{Cl}$. No differences are observed with the NT3 mutation relative to WT. All data are the mean \pm SEM of $n=5-20$ coverslips from at least two independent cultures with at least 20 synapses analyzed per coverslip.

NT3 VGLUT1-pH presumably depends on the C-terminal dileucine-like motif, this suggests that the C-terminal signal depends more strongly on AP-2 than the N-terminal signals. Consistent with this, the poststimulus endocytosis of FV/GG VGLUT1-pH, which depends primarily on the N-terminal dileucine-like motifs, was not influenced by AP-2 knockdown (Fig. 8D). AP-2 thus appears more important for VGLUT1 endocytosis mediated by the C-terminal dileucine-like motif, suggesting recognition of the $\mathrm{N}$-terminal motifs primarily by other adaptors. Indeed, the closely related adaptor AP-1 plays a role in synaptic vesicle recycling (Glyvuk et al., 2010; Cheung and Cousin, 2012).

AP-1 knockdown has different effects on the behavior of VGLUT1-pH. Relative to vector control, knockdown of AP-1 $\gamma$ does not significantly alter the poststimulus endocytic rate of WT, NT3, or FV/GG VGLUT1-pH (Fig. 8B-D). However, the peak level of FV/GG VGLUT1-pH fluorescence during stimulation is lower with AP-1 knockdown relative to vector control (Fig. $8 D)$. This effect is specific for FV/GG VGLUT1-pH since AP-1 knockdown has no significant effect on the response to stimulation of either WT or NT3 (Fig. 8B,C). Experiments using a second shRNA targeting AP-1 $\gamma$ (AP-1B shRNA) confirm the reduced peak response of FV/GG VGLUT1-pH to stimulation, arguing against an off-target effect (Fig. 8D). Quantification of the peak fluorescence as a fraction of the total internal pool of transporters confirms the decrease in response to stimulation (vector, $65.2 \pm 1.8 \%$; AP-1A, $55.0 \pm 1.6 \%$; AP-1B, $52.7 \pm 4.8 \%$; $p<0.05$ for both comparisons to control) (Fig. $8 E$ ). To determine whether the decreased peak fluorescence represents a decrease in exocytosis or an increase in endocytosis during stimulation, we stimulated cells in the presence of the $\mathrm{H}^{+}$-ATPase inhibitor bafilomycin. The fraction of VGLUT1-pH that responds to stimulation at $30 \mathrm{~Hz}$ for $1 \mathrm{~min}$ is significantly smaller in cells depleted of AP-1 (vector, $68.3 \pm$ 2.1\%; AP-1A, $52.4 \pm 3.1 \%$; $p<0.0006$ ) (Fig. $8 F$ ). Together, the results show that AP-2 acts via the C-terminal dileucinelike signal to promote endocytosis of VGLUT1, whereas AP-1 acts via the $\mathrm{N}$-terminal motifs to influence the proportion of VGLUT1 in the recycling pool.

\section{VGLUT1 and VGLUT2 differ in their trafficking}

Several lines of evidence have converged to suggest differences in the trafficking of VGLUT1 and VGLUT2. Despite the loss of VGLUT1 in knock-out mice, glutamate release persists at hippocampal synapses expressing VGLUT2, but the properties of release differ (Fremeau et al., 2004b; Wojcik et al., 2004). Reflecting the activity of VGLUT2, the knock-out synapses exhibit increased short-term depression in response to high-frequency stimulation compared to the predominantly VGLUT1 ${ }^{+}$ synapses of WT, consistent with a higher release probability at $\mathrm{VGLUT}^{+}$than VGLUT1 $^{+}$synapses. The recent evidence that VGLUT1 and VGLUT2 can control release probability further supports differences in their trafficking (Weston et al., 2011). To determine whether VGLUT1 and VGLUT2 do in fact recycle differently, we inserted ecliptic pHluorin into the large luminal loop of VGLUT2 in a position similar to the insertion site in VGLUT1 (Voglmaier et al., 2006). We then compared the response of WT VGLUT1-pH and VGLUT2-pH to stimulation in transfected hippocampal neurons at $40 \mathrm{~Hz}$ for $1 \mathrm{~min}$. Initial examination reveals that the kinetics of VGLUT2 recycling appear different (Fig. 9A). Quantification confirms that the decline in fluorescence from peak levels by the end of stimulation is smaller for VGLUT2 (VGLUT1, $55.8 \pm 3.2 \%$; VGLUT2, $38.2 \pm 2.6 \%$ of peak fluorescence; $p<0.0006)$. The rate of poststimulus endocytosis also appears slower for VGLUT2 (VGLUT1, $\tau_{\text {decay }}=31.2 \pm 1.9 \mathrm{~s}$; VGLUT2, $\tau_{\text {decay }}=41.9 \pm 5.9 \mathrm{~s} ; p=0.12$ ) (Fig. 9A). Thus, VGLUT1 and VGLUT2 exhibit differences in recycling.

The N- and C-terminal sequences of VGLUT1 and VGLUT2 diverge considerably, but VGLUT2 contains the C-terminal dileucine-like motif $\left({ }_{512}\right.$ SEEKCGFI $\left._{519}\right)$ conserved in the other VGLUTs. However, the N-terminal dileucine-like motifs of VGLUT1 do not appear to be well conserved in VGLUT2 or VGLUT3. To assess the importance of the C-terminal dileucine-like signal in VGLUT2, we replaced Phe-518 and Ile519 with glycine (FI/GG) and introduced these mutations into VGLUT2-pH, analogous to the FV/GG mutation in VGLUT1. Transfected into neurons, like WT VGLUT1-pH, the fluorescence of WT VGLUT2-pH is barely detectable at rest, but $\mathrm{NH}_{4} \mathrm{Cl}$ reveals considerable fluorescence, indicating expression of the protein in an acidic, intracellular compartment (Fig. 9B). In contrast, FI/GG VGLUT2 exhibits substantial steady-state fluorescence at $\mathrm{pH} 7.4$ that is quenchable at $\mathrm{pH} 5.5$, indicating mislocalization of the mutant to the plasma membrane. Quantification confirms that the FI/GG mutation increases steady-state surface levels of VGLUT2-pH >10-fold (WT, $2.4 \pm 0.3 \%$; FI/GG, $29.7 \pm 1.8 \% ; p<0.0001)$, compared to a less than threefold increase observed with the FV/GG mutation in VGLUT1 (Figs. 

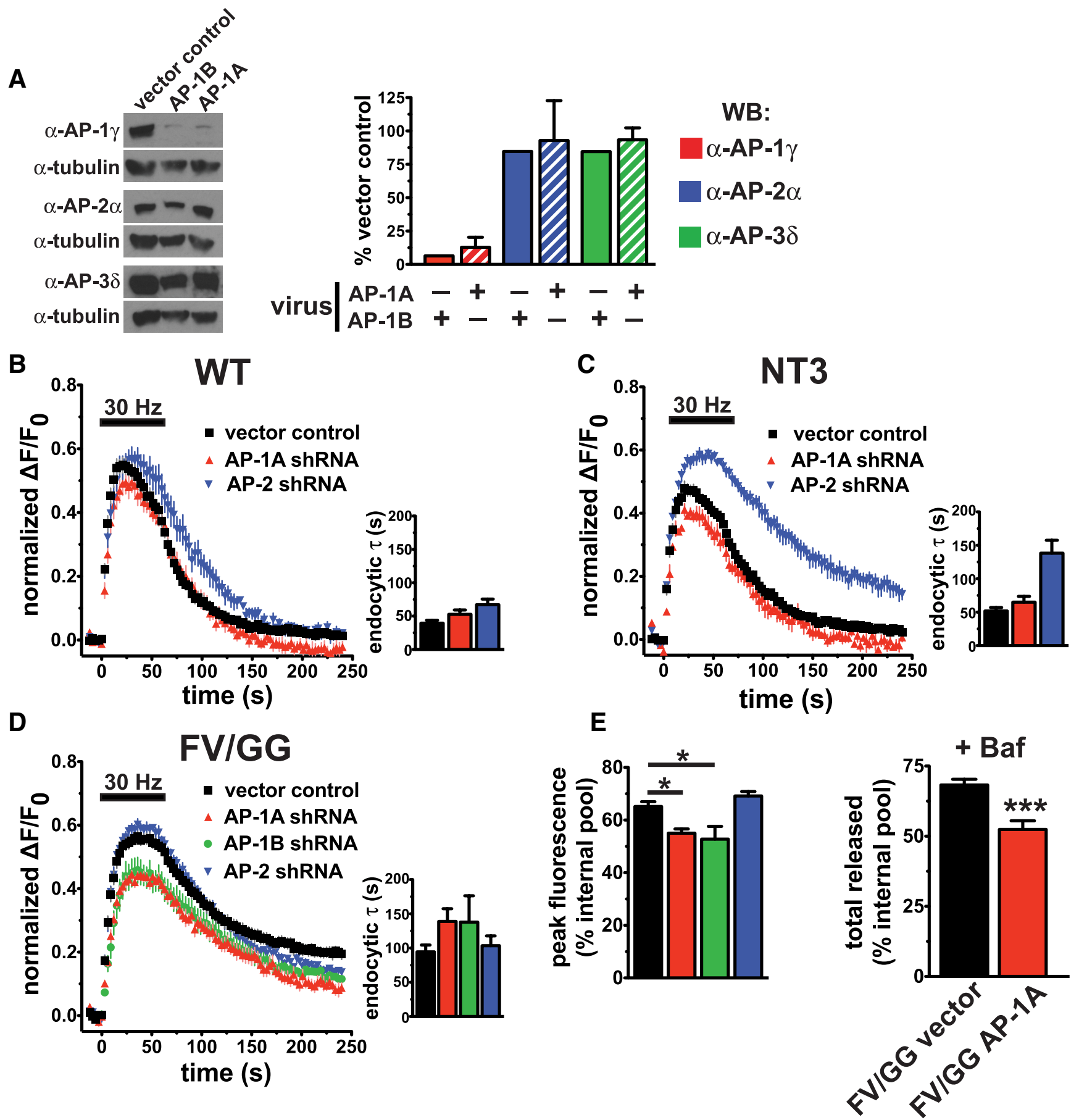

Figure 8. $\mathrm{N}$ - and C-terminal dileucine-like motifs use different clathrin adaptor proteins. $\boldsymbol{A}$, Western blotting of cell lysates from primary hippocampal neurons infected with lentiviral particles containing shRNA constructs demonstrates that only AP-1 levels are reduced by the AP-1A and AP-1B hairpins. The graph shows quantification of AP protein levels normalized to tubulin controls. Data are presented as percentages of vector controls. B, Time course of fluorescence changes in neurons transfected with WT VGLUT1-pH and infected with viral particles expressing empty vector (black), AP-1A shRNA (red), or AP-2 shRNA (blue), during and after stimulation at $30 \mathrm{~Hz}$ for $1 \mathrm{~min}$ (bar). Each trace was normalized to the size of the total pool of VGLUT1-pH, determined by $\mathrm{NH}_{4} \mathrm{Cl}$ application. Knockdown of AP-2 (blue), but not AP-1 (red), significantly slows poststimulus endocytosis of WT VGLUT1-pH (vector control, $\tau_{\text {decay }}=39.5 \pm 4.2 \mathrm{~s} ; \mathrm{AP}-1 \mathrm{~A}, \tau_{\text {decay }}=52.6 \pm 6.6 \mathrm{~s} ; \mathrm{AP}-2, \tau_{\text {decay }}=67.1 \pm 8.5 \mathrm{~s}$; compared to vector control, AP-2, $p<0.05 ;$ AP-1A, $p>0.05$; one-way ANOVA, Tukey's post-test). C, Depletion of AP-2, but not AP-1, also significantly slows poststimulus endocytosis of NT3 VGLUT1-pH [vector control (black), $\tau_{\text {decay }}=51.7 \pm 5.2 \mathrm{~s} ; \mathrm{AP}-1 \mathrm{~A}$ (red), $\tau_{\text {decay }}=64.9 \pm 8.8 \mathrm{~s}$; AP-2 (blue), $\tau_{\text {decay }}=138.2 \pm 19.4$ s; compared to vector control, AP-2, $p<0.001 ;$ AP-1A, $p>0.05$; one-way ANOVA, Tukey's post-test]. $D$, The rate of poststimulus endocytosis of FV/GG VGLUT1-pH was not altered by knockdown of either AP-1 or AP-2 [vector control (black), $\tau_{\text {decay }}=94.4 \pm 10.0 \mathrm{~s} ; \mathrm{AP}-1 \mathrm{~A}$ (red), $\tau_{\text {decay }}=138.9 \pm 18.5 \mathrm{~s} ; \mathrm{AP}-1 \mathrm{~B}$ (green), $\tau_{\text {decay }}=$ $137.9 \pm 38.5 \mathrm{~s}$; AP-2 (blue), $\tau_{\text {decay }}=103.4 \pm 14.4 \mathrm{~s} ; p>0.05$ for all comparisons; one-way ANOVA, Tukey's post-test]. $E$, Knockdown of AP-1, but not AP-2, lowers the peak fluorescence of FV/GG VGLUT1-pH during stimulation, normalized to the total internal pool [vector control (black), $65.2 \pm 1.8 \%$; AP-1A (red), $55.0 \pm 1.6 \%$; AP-1B (green), $52.7 \pm 4.8 \%$; AP-2 (blue), $69.2 \pm 1.7 \%$; compared to vector control, AP- $1 \mathrm{~A},{ }^{*} p<0.05 ; \mathrm{AP}-1 \mathrm{~B},{ }^{*} p<0.05 ; \mathrm{AP}-2, p>0.05$; one-way ANOVA, Tukey's post-test]. $\boldsymbol{F}$, Stimulation in the presence of the $\mathrm{H}^{+}$-ATPase inhibitor, bafilomycin, reveals that AP-1 knockdown significantly decreases the fraction of the total internal pool of FV/GG VGLUT1-pH released with a $30 \mathrm{~Hz} 1$ min stimulus [vector control (black), $68.3 \pm 2.1 \%$; AP-1A (red), $52.4 \pm 3.1 \%$; *** $p=0.0006$; unpaired, two-tailed $t$ test]. Data are shown as the mean \pm SEM. For $\boldsymbol{B}-\boldsymbol{F}, n=5-20$ coverslips from at least two independent cultures with at least 20 synapses analyzed per coverslip. 

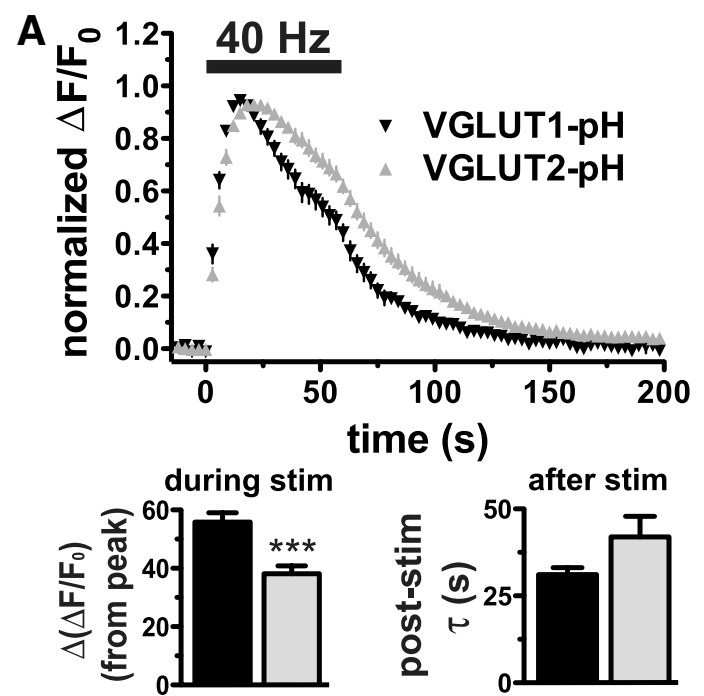

B
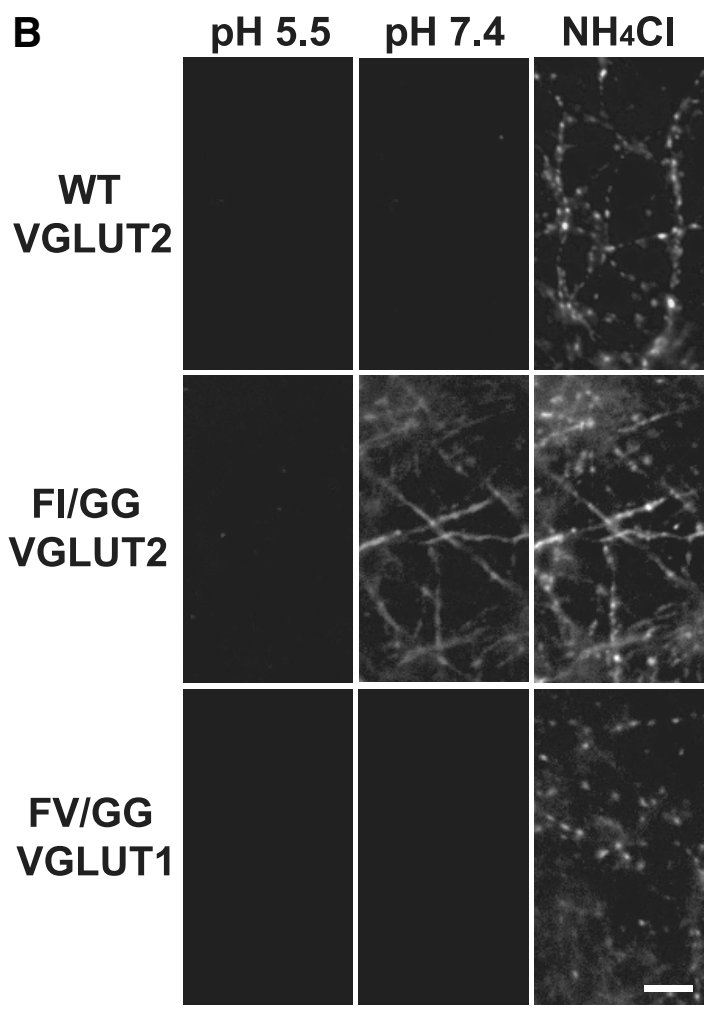

C

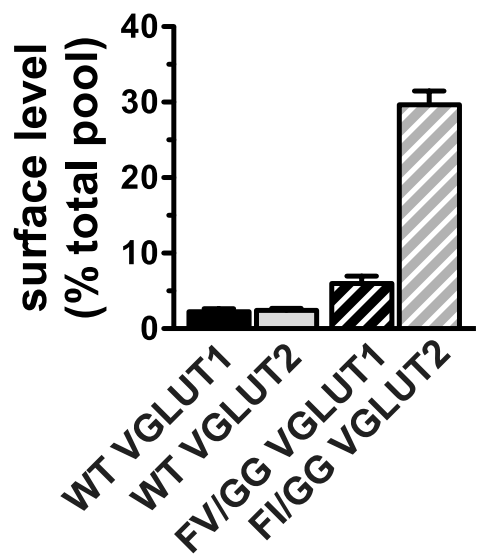

Figure 9. VGLUT1 and VGLUT2 differ in their trafficking. $\boldsymbol{A}$, Time course of fluorescence changes in neurons transfected with either WT VGLUT1-pH (black) or VGLUT2-pH (gray) to a 40 $\mathrm{Hz}, 1$ min stimulation (bar). Traces were normalized to peak fluorescence during stimulation.
$2 C, 9 C)$. Thus, while VGLUT2 depends almost exclusively on the C-terminal dileucine-like signal for endocytosis and synaptic targeting, VGLUT1 also relies on its N-terminal trafficking motifs.

\section{Discussion}

Despite the strong conservation of a C-terminal dileucine-like motif in all three VGLUTs, mutation of this motif in VGLUT1 fails to eliminate synaptic targeting and compensatory endocytosis. Indeed, deletion of the entire $\mathrm{C}$ terminus does not abolish synaptic targeting and response to stimulation, although we demonstrated previously that this region is sufficient to confer internalization to a plasma membrane protein (Voglmaier et al., 2006). We now find that the large cytosolic $N$ terminus of VGLUT1 contains two additional, previously unidentified dileucine-like motifs. The N terminus of VGLUT1 can alone mediate internalization of the plasma membrane protein Tac, and this depends on key residues in the atypical dileucine-like motifs. Examining these motifs in the context of the full VGLUT1 sequence, we found that mutation of both N-terminal motifs (NT3) fails to influence the recycling kinetics of VGLUT1-pH over a range of stimulation frequencies $(5-80 \mathrm{~Hz})$, including the sustained high-frequency stimulation associated with activitydependent bulk endocytosis (Clayton et al., 2008). The NT3 mutation also fails to alter the rate of VGLUT1 exocytosis in response to $10 \mathrm{~Hz}$ stimulation. In addition, loss of either the $\mathrm{N}$ - or C-terminal motif does not influence the size of the readily releasable and recycling synaptic vesicle pools of VGLUT1. However, deletion of these motifs in both termini essentially eliminates synaptic targeting of VGLUT1 and its response to stimulation. Thus, the minimal effect of N-terminal mutations on VGLUT1 endocytosis likely reflects redundancy with the C-terminal dileucine-like signal.

Despite the absence of a detectable effect on compensatory endocytosis, mutation of the N-terminal motifs triples the amount of transporter present on the cell surface at rest. This effect requires mutation of both $\mathrm{N}$-terminal motifs, suggesting redundancy. Although mutation of the C-terminal motif produces a substantial defect in poststimulus compensatory endocytosis, it increases steady-state surface expression of VGLUT1 to the same extent. Thus, different mechanisms may regulate steady-state surface expression and compensatory endocytosis. The results demonstrate that despite their differences in compensatory endocytosis, either the two $\mathrm{N}$-terminal motifs or the

The extent of fluorescence decay from peak fluorescence $\left[\Delta\left(\Delta F / F_{0}\right)\right]$ during stimulation is greater for VGLUT1 than VGLUT2 (VGLUT1, $55.8 \pm 3.2 \%$ of peak $F$; VGLUT2, $38.2 \pm 2.6 \%$ of peak $F$; ${ }^{* * *} p<0.0006$; unpaired, two-tailed $t$ test; bottom left inset). The rate of VGLUT2 poststimulus endocytosis is not significantly slower than that for VGLUT1 (VGLUT1, $\tau_{\text {decay }}=$ $31.2 \pm 1.9 \mathrm{~s} ;$ VGLUT2, $\tau_{\text {decay }}=41.9 \pm 5.9 \mathrm{~s} ; p=0.12$; unpaired, two-tailed $t$ test; bottom right inset). For all graphs, $n=8-9$ coverslips from four independent cultures with at least 20 synapses analyzed per coverslip. $\boldsymbol{B}$, Hippocampal neurons transfected with WT VGLUT2-pH FI/GG VGLUT2-pH, or FV/GG VGLUT1-pH were imaged in Tyrode's solution buffered with MES to pH 5.5 to quench surface fluorescence, at rest (pH 7.4), and upon alkalinization in $\mathrm{NH}_{4} \mathrm{Cl}$ to measure total fluorescence. Significant fluorescence is observed with FI/GG VGLUT2-pH at rest (pH 7.4) that is quenched by pH 5.5 Tyrode's solution, indicating that a large amount of the transporter is present on the cell surface at rest. $C$, At rest, the cell surface expression of WT VGLUT1-pH ( $2.2 \pm 0.4 \%$ of total protein) and WT VGLUT2-pH ( $2.4 \pm 0.3 \%$ of total protein) are similar ( $p=0.65$; unpaired, two-tailed $t$ test). In contrast, considerably more FI/GG VGLUT2-pH is on the cell surface at rest than FV/GG VGLUT1-pH (FV/GG, $6.0 \pm 1.0 \%$; Fl/GG, $29.7 \pm 1.8 \%$; $p<0.0001$, unpaired, two-tailed $t$ test). $n=5-15$ coverslips from at least four independent cultures with at least 20 synapses analyzed per coverslip. Data for WT and FV/GG VGLUT1-pH are from Figure 2C. Data are shown as mean \pm SEM. Scale bar, $10 \mu \mathrm{m}$. 
C-terminal motif suffice for VGLUT1's trafficking to synapses and incorporation into synaptic vesicles.

Isolating the $\mathrm{N}$ and $\mathrm{C}$ termini through mutation of key residues in either VGLUT1 region, combined with shRNA-mediated knockdown of AP-1 and AP-2, uncovered two distinct mechanisms mediated by the $\mathrm{N}$ - and $\mathrm{C}$-terminal dileucine-like motifs. In the absence of a functional N-terminal motif (NT3 VGLUT1$\mathrm{pH}), \mathrm{AP}-2$ depletion has a much stronger effect on endocytosis than it does with the WT transporter. Since NT3 VGLUT1-pH relies on the C-terminal motif for endocytosis, this motif must depend primarily on AP-2, consistent with the role of AP-2 and this motif in compensatory endocytosis (Dittman and Ryan, 2009; Kim and Ryan, 2009; Royle and Lagnado, 2010). In contrast, AP-2 depletion has no additional effect on FV/GG VGLUT1 endocytosis. Since FV/GG depends on the N-terminal motifs for internalization, these motifs do not use AP-2 or can use alternative adaptors such as AP-1 or AP-3. AP-2 binds PIP2, helping to localize the adaptor at the plasma membrane, where it retrieves synaptic vesicle components. Although the role of AP-1 is best established in protein trafficking at the trans-Golgi network (TGN) and endosomes, it also localizes to the nerve terminal (Glyvuk et al., 2010), where it associates with synaptic vesicles (Takamori et al., 2006) and contributes to their recycling through endosomal intermediates. Knock-out of a brain-enriched isoform of the small AP- 1 subunit (AP- $\sigma 1 \mathrm{~B}$ ) or knockdown of AP-1 impairs synaptic vesicle reformation from the large membrane structures that appear with strong stimulation (Glyvuk et al., 2010; Cheung and Cousin, 2012). Arf1, which helps to localize AP-1 at membranes (Traub et al., 1993), is also required for synaptic vesicle reformation from endosomes (Faúndez et al., 1997). VGLUT1 may localize to these structures by direct fusion of a plasma membrane derived vesicle (Heuser and Reese, 1973; Wucherpfennig et al., 2003; Sudhof, 2004; Rizzoli et al., 2006; Hoopmann et al., 2010) or by activity-dependent bulk endocytosis (Cousin, 2009). AP-1 may also generate VGLUT1 containing vesicles directly from the plasma membrane. Although Arf1 localizes primarily to TGN and endosomal membranes, it also occurs on the presynaptic plasma membrane (Morciano et al., 2005; Morciano et al., 2009) and has been reported to promote dynamin-independent endocytosis in nonneuronal cells (Kumari and Mayor, 2008). Alternatively, VGLUT1 may engage an AP-independent mechanism for internalization of the transporter. Previous work has indeed shown that impairment of endocytosis with AP-2 depletion can be partially rescued by pharmacological inhibition of AP-1 and/or AP-3 (Polo-Parada et al., 2001; Voglmaier et al., 2006; Kim and Ryan, 2009).

In contrast to AP-2, depletion of AP-1 has no effect on poststimulus endocytosis of WT or NT3 VGLUT1-pH. The C-terminal dileucine motif thus does not seem to interact functionally with AP-1. Interestingly, AP-1 knockdown reduces the peak fluorescence reached during stimulation of $\mathrm{FV} / \mathrm{GG}$ VGLUT1-pH. Moreover, stimulation in the presence of the $\mathrm{H}^{+}$ATPase inhibitor bafilomycin indicates a reduction in recycling pool size. Thus, the N-terminal motifs target VGLUT1 to the recycling pool in an AP-1-dependent manner. Differences in the endocytic pathway traveled by synaptic proteins such as VGLUT1 could translate into changes in release probability and synaptic plasticity in several ways. First, recycling kinetics may simply determine how quickly vesicles can be reused (Fernández-Alfonso and Ryan, 2004; Sudhof, 2004; Ertunc et al., 2007). Synaptic vesicle reformation directly from the plasma membrane via AP-2 may occur more rapidly than reformation by AP- 1 from endosomelike compartments. Second, different APs may clear plasma mem- brane release sites with different efficiencies, therefore affecting exocytosis (Hosoi et al., 2009; Neher, 2010). Third, different pathways could direct vesicles to different functional pools with differing probabilities of release, which may or may not be present in different locations (Rizzoli and Betz, 2004, 2005; Vanden Berghe and Klingauf, 2006). Finally, independent sorting of vesicle components by APs could produce vesicles with varying protein compositions that underlie their different functional properties (Bonanomi et al., 2006; Voglmaier and Edwards, 2007).

In contrast to the conserved C-terminal dileucine-like signal, the N-terminal motifs do not appear to be well conserved in VGLUT2 or VGLUT3. Interestingly, analogous mutation of the C-terminal motif has a much larger effect on VGLUT2 than VGLUT1: replacement by glycine in VGLUT1 (FV/GG mutant) increases the steady-state cell surface fraction of the transporter threefold, but the FI/GG mutation increases surface expression of VGLUT2 by 10 -fold. VGLUT1 thus relies on multiple trafficking signals, and VGLUT2 appears largely dependent on only one, predicting differences in the recycling of these proteins at the nerve terminal. Indeed, analysis of pHluorin fusions shows that the two proteins differ in response to stimulation. During stimulation, when endocytosis begins to reduce the peak fluorescence achieved by exocytosis, VGLUT1-pH shows a more rapid fluorescence decline than VGLUT2-pH, suggesting less efficient retrieval of VGLUT2 than VGLUT1. Presumably, the additional $\mathrm{N}$-terminal dileucine motifs in VGLUT1 contribute to these observed differences.

The question remains as to how the observed differences in VGLUT1 and VGLUT2 trafficking influence transmitter release. Isoform expression correlates with the probability of release, and a direct comparison of VGLUT1 ${ }^{+}$and VGLUT2 ${ }^{+}$synapses using knock-out mice has shown differences in short-term plasticity (Fremeau et al., 2004b). More recently, heterologous expression in VGLUT-lacking neurons has demonstrated that the two isoforms can themselves influence the probability of transmitter release, suggesting a direct effect on vesicle release properties rather than simply a correlation (Weston et al., 2011). However, VGLUT1 and VGLUT2 exhibit similar transport activity, suggesting that another mechanism must account for the differences in release (Fremeau et al., 2001; Takamori, 2006).

We hypothesize that the role of the VGLUT isoform in glutamate release reflects their targeting to synaptic vesicles with different properties. Previously, we showed that VGLUT1 interacts with the endocytic protein endophilin via a polyproline domain not found in VGLUT2 or VGLUT3 (De Gois et al., 2006; Vinatier et al., 2006; Voglmaier et al., 2006). A mutation disrupting this interaction converts the properties of release from a VGLUT1 ${ }^{+}$ to VGLUT2 ${ }^{+}$synapse (Weston et al., 2011). However, the effect of the polyproline interaction with endophilin depends on the C-terminal dileucine-like motif (Voglmaier et al., 2006). In particular, the interaction with endophilin directs VGLUT1 toward an endocytic pathway dependent on this motif, and away from one involving AP-1 or AP-3 (Voglmaier et al., 2006). Since we now find that the $\mathrm{C}$-terminal dileucine-like motif depends on AP-2 and the N-terminal motifs on AP-1, the interaction with endophilin appears to influence the endocytic pathway used, which may correspond to recycling directly from the plasma membrane versus through an endosomal or cisternal intermediate. Multiple dileucine-like motifs thus endow VGLUT1 with the capacity for faster recycling and the potential for multiple recycling pathways that may in turn contribute to plasticity. In contrast, VGLUT2 appears to primarily rely upon a single 
dileucine-like motif, perhaps accounting for its slower recycling and expression at synapses with lower potential for plasticity.

\section{References}

Aihara Y, Mashima H, Onda H, Hisano S, Kasuya H, Hori T, Yamada S, Tomura H, Yamada Y, Inoue I, Kojima I, Takeda J (2000) Molecular cloning of a novel brain-type $\mathrm{Na}(+)$-dependent inorganic phosphate cotransporter. J Neurochem 74:2622-2625. Medline

Ariel P, Ryan TA (2010) Optical mapping of release properties in synapses. Front Neural Circuits Aug 12; 4.

Bai L, Xu H, Collins JF, Ghishan FK (2001) Molecular and functional analysis of a novel neuronal vesicular glutamate transporter. J Biol Chem 276:36764-36769. CrossRef Medline

Bellocchio EE, Reimer RJ, Fremeau RT Jr, Edwards RH (2000) Uptake of glutamate into synaptic vesicles by an inorganic phosphate transporter. Science 289:957-960. CrossRef Medline

Bonanomi D, Benfenati F, Valtorta F (2006) Protein sorting in the synaptic vesicle life cycle. Prog Neurobiol 80:177-217. CrossRef Medline

Bonifacino JS, Traub LM (2003) Signals for sorting of transmembrane proteins to endosomes and lysosomes. Annu Rev Biochem 72:395-447. CrossRef Medline

Bonifacino JS, Cosson P, Klausner RD (1990) Colocalized transmembrane determinants for ER degradation and subunit assembly explain the intracellular fate of TCR chains. Cell 63:503-513. CrossRef Medline

Cheung G, Cousin MA (2012) Adaptor protein complexes 1 and 3 are essential for generation of synaptic vesicles from activity-dependent bulk endosomes. J Neurosci 32:6014-6023. CrossRef Medline

Clayton EL, Evans GJ, Cousin MA (2008) Bulk synaptic vesicle endocytosis is rapidly triggered during strong stimulation. J Neurosci 28:6627-6632. CrossRef Medline

Cousin MA (2009) Activity-dependent bulk synaptic vesicle endocytosis-a fast, high capacity membrane retrieval mechanism. Mol Neurobiol 39: 185-189. CrossRef Medline

Das SS, Banker GA (2006) The role of protein interaction motifs in regulating the polarity and clustering of the metabotropic glutamate receptor mGluR1a. J Neurosci 26:8115-8125. CrossRef Medline

De Gois S, Jeanclos E, Morris M, Grewal S, Varoqui H, Erickson JD (2006) Identification of endophilins 1 and 3 as selective binding partners for VGLUT1 and their co-localization in neocortical glutamatergic synapses: implications for vesicular glutamate transporter trafficking and excitatory vesicle formation. Cell Mol Neurobiol 26:679-693. Medline

Dittman J, Ryan TA (2009) Molecular circuitry of endocytosis at nerve terminals. Annu Rev Cell Dev Biol 25:133-160. CrossRef Medline

Dugast M, Toussaint H, Dousset C, Benaroch P (2005) AP2 clathrin adaptor complex, but not AP1, controls the access of the major histocompatibility complex (MHC) class II to endosomes. J Biol Chem 280:19656-19664. CrossRef Medline

Ertunc M, Sara Y, Chung C, Atasoy D, Virmani T, Kavalali ET (2007) Fast synaptic vesicle reuse slows the rate of synaptic depression in the CA1 region of hippocampus. J Neurosci 27:341-354. CrossRef Medline

Faúndez V, Horng JT, Kelly RB (1997) ADP ribosylation factor 1 is required for synaptic vesicle budding in PC12 cells. J Cell Biol 138:505-515. CrossRef Medline

Fernández-Alfonso T, Ryan TA (2004) The kinetics of synaptic vesicle pool depletion at CNS synaptic terminals. Neuron 41:943-953. CrossRef Medline

Fremeau RT Jr, Troyer MD, Pahner I, Nygaard GO, Tran CH, Reimer RJ, Bellocchio EE, Fortin D, Storm-Mathisen J, Edwards RH (2001) The expression of vesicular glutamate transporters defines two classes of excitatory synapse. Neuron 31:247-260. CrossRef Medline

Fremeau RT Jr, Burman J, Qureshi T, Tran CH, Proctor J, Johnson J, Zhang H, Sulzer D, Copenhagen DR, Storm-Mathisen J, Reimer RJ, Chaudhry FA, Edwards RH (2002) The identification of vesicular glutamate transporter 3 suggests novel modes of signaling by glutamate. Proc Natl Acad Sci U S A 99:14488-14493. CrossRef Medline

Fremeau RT Jr, Voglmaier S, Seal RP, Edwards RH (2004a) VGLUTs define subsets of excitatory neurons and suggest novel roles for glutamate. Trends Neurosci 27:98-103. CrossRef Medline

Fremeau RT Jr, Kam K, Qureshi T, Johnson J, Copenhagen DR, StormMathisen J, Chaudhry FA, Nicoll RA, Edwards RH (2004b) Vesicular glutamate transporters 1 and 2 target to functionally distinct synaptic release sites. Science 304:1815-1819. CrossRef Medline
Geisler C, Dietrich J, Nielsen BL, Kastrup J, Lauritsen JP, Odum N, Christensen MD (1998) Leucine-based receptor sorting motifs are dependent on the spacing relative to the plasma membrane. J Biol Chem 273:2131621323. CrossRef Medline

Glyvuk N, Tsytsyura Y, Geumann C, D’Hooge R, Hüve J, Kratzke M, Baltes J, Böning D, Klingauf J, Schu P (2010) AP-1/sigma1B-adaptin mediates endosomal synaptic vesicle recycling, learning and memory. EMBO J 29: 1318-1330. CrossRef Medline

Gras C, Herzog E, Bellenchi GC, Bernard V, Ravassard P, Pohl M, Gasnier B, Giros B, El Mestikawy S (2002) A third vesicular glutamate transporter expressed by cholinergic and serotoninergic neurons. J Neurosci 22:54425451. Medline

Hawryluk MJ, Keyel PA, Mishra SK, Watkins SC, Heuser JE, Traub LM (2006) Epsin 1 is a polyubiquitin-selective clathrin-associated sorting protein. Traffic 7:262-281. CrossRef Medline

He S, Sun JM, Li L, Davie JR (2005) Differential intranuclear organization of transcription factors Sp1 and Sp3. Mol Biol Cell 16:4073-4083. CrossRef Medline

Herzog E, Bellenchi GC, Gras C, Bernard V, Ravassard P, Bedet C, Gasnier B, Giros B, El Mestikawy S (2001) The existence of a second vesicular glutamate transporter specifies subpopulations of glutamatergic neurons. J Neurosci 21:RC181. Medline

Heuser JE, Reese TS (1973) Evidence for recycling of synaptic vesicle membrane during transmitter release at the frog neuromuscular junction. J Cell Biol 57:315-344. CrossRef Medline

Hoopmann P, Punge A, Barysch SV, Westphal V, Bückers J, Opazo F, Bethani I, Lauterbach MA, Hell SW, Rizzoli SO (2010) Endosomal sorting of readily releasable synaptic vesicles. Proc Natl Acad Sci U S A 107:1905519060. CrossRef Medline

Hosoi N, Holt M, Sakaba T (2009) Calcium dependence of exo- and endocytotic coupling at a glutamatergic synapse. Neuron 63:216-229. CrossRef Medline

Hua Z, Leal-Ortiz S, Foss SM, Waites CL, Garner CC, Voglmaier SM, Edwards RH (2011) v-SNARE composition distinguishes synaptic vesicle pools. Neuron 71:474-487. CrossRef Medline

Kantheti P, Qiao X, Diaz ME, Peden AA, Meyer GE, Carskadon SL, Kapfhamer D, Sufalko D, Robinson MS, Noebels JL, Burmeister M (1998) Mutation in AP-3 delta in the mocha mouse links endosomal transport to storage deficiency in platelets, microsomes and synaptic vesicles. Neuron 21:111-122. CrossRef Medline

Kim SH, Ryan TA (2009) Synaptic vesicle recycling at CNS snapses without AP-2. J Neurosci 29:3865-3874. CrossRef Medline

Kozak M (1986) Point mutations define a sequence flanking at the AUG initiator codon that modulates translation by eukaryotic ribosomes. Cell 44:283-292. CrossRef Medline

Kumari S, Mayor S (2008) ARF1 is directly involved in dynaminindependent endocytosis. Nat Cell Biol 10:30-41. CrossRef Medline

Li H, Foss SM, Dobryy YL, Park CK, Hires SA, Shaner NC, Tsien RY, Osborne LC, Voglmaier SM (2011) Concurrent imaging of synaptic vesicle recycling and calcium dynamics. Front Mol Neurosci 4:34. Medline

Lois C, Hong EJ, Pease S, Brown EJ, Baltimore D (2002) Germline transmission and tissue-specific expression of transgenes delivered by lentiviral vectors. Science 295:868-872. CrossRef Medline

Meyer C, Zizioli D, Lausmann S, Eskelinen EL, Hamann J, Saftig P, von Figura K, Schu P (2000) mu1A-adaptin-deficient mice: lethality, loss of AP-1 binding and rerouting of mannose 6-phosphate receptors. EMBO J 19: 2193-2203. CrossRef Medline

Miesenböck G, De Angelis DA, Rothman JE (1998) Visualizing secretion and synaptic transmission with $\mathrm{pH}$-sensitive green fluorescent proteins. Nature 394:192-195. CrossRef Medline

Morciano M, Burré J, Corvey C, Karas M, Zimmermann H, Volknandt W (2005) Immunoisolation of two synaptic vesicle pools from synaptosomes: a proteomics analysis. J Neurochem 95:1732-1745. CrossRef Medline

Morciano M, Beckhaus T, Karas M, Zimmermann H, Volknandt W (2009) The proteome of the presynaptic active zone: from docked synaptic vesicles to adhesion molecules and maxi-channels. J Neurochem 108:662675. CrossRef Medline

Motley A, Bright NA, Seaman MN, Robinson MS (2003) Clathrin-mediated endocytosis in AP-2-depleted cells. J Cell Biol 162:909-918. CrossRef Medline 
Neher E (2010) What is rate-limiting during sustained synaptic activity: vesicle supply or release sites? Front Synaptic Neurosci 2:1-6. Medline

Omote H, Miyaji T, Juge N, Moriyama Y (2011) Vesicular neurotransmitter transporter: bioenergetics and regulation of glutamate transport. Biochemistry 50:5558-5565. CrossRef Medline

Owen DJ, Collins BM, Evans PR (2004) Adaptors for clathrin coats: structure and function. Annu Rev Cell Dev Biol 20:153-191. CrossRef Medline

Peden AA, Park GY, Scheller RH (2001) The Di-leucine motif of vesicleassociated membrane protein 4 is required for its localization and AP-1 binding. J Biol Chem 276:49183-49187. CrossRef Medline

Polo-Parada L, Bose CM, Landmesser LT (2001) Alterations in transmission, vesicle dynamics, and transmitter release machinery at NCAMdeficient neuromuscular junctions. Neuron 32:815-828. CrossRef Medline

Rizzoli SO, Betz WJ (2004) The structural organization of the readily releasable pool of synaptic vesicles. Science 303:2037-2039. CrossRef Medline

Rizzoli SO, Betz WJ (2005) Synaptic vesicle pools. Nat Rev Neurosci 6:5769. CrossRef Medline

Rizzoli SO, Bethani I, Zwilling D, Wenzel D, Siddiqui TJ, Brandhorst D, Jahn R (2006) Evidence for early endosome-like fusion of recently endocytosed synaptic vesicles. Traffic 7:1163-1176. CrossRef Medline

Royle SJ, Lagnado L (2010) Clathrin-mediated endocytosis at the synaptic terminal: bridging the gap between physiology and molecules. Traffic 11:1489-1497. CrossRef Medline

Sankaranarayanan S, Ryan TA (2000) Real-time measurements of vesicleSNARE recycling in synapses of the central nervous system. Nat Cell Biol 2:197-204. CrossRef Medline

Sankaranarayanan S, Ryan TA (2001) Calcium accelerates endocytosis of vSNAREs at hippocampal synapses. Nat Neurosci 4:129-136. CrossRef Medline

Schäfer MK, Varoqui H, Defamie N, Weihe E, Erickson JD (2002) Molecular cloning and functional identification of mouse vesicular glutamate transporter 3 and its expression in subsets of novel excitatory neurons. J Biol Chem 277:50734-50748. CrossRef Medline

Steenhuis P, Herder S, Gelis S, Braulke T, Storch S (2010) Lysosomal targeting of the CLN7 membrane glycoprotein and transport via the plasma membrane require a dileucine motif. Traffic 11:987-1000. CrossRef Medline

Sudhof TC (2004) The synaptic vesicle cycle. Annu Rev Neurosci 27:509547. CrossRef Medline

Takamori S (2006) VGLUTs: 'exciting' times for glutamatergic research? Neurosci Res 55:343-351. CrossRef Medline

Takamori S, Rhee JS, Rosenmund C, Jahn R (2000) Identification of a vesicular glutamate transporter that defines a glutamatergic phenotype in neurons. Nature 407:189-194. CrossRef Medline

Takamori S, Rhee JS, Rosenmund C, Jahn R (2001) Identification of differentiation-associated brain-specific phosphate transporter as a second vesicular glutamate transporter. J Neurosci 21:RC182. Medline

Takamori S, Malherbe P, Broger C, Jahn R (2002) Molecular cloning and functional characterization of human vesicular glutamate transporter 3 . EMBO Rep 3:798-803. CrossRef Medline

Takamori S, Holt M, Stenius K, Lemke EA, Grønborg M, Riedel D, Urlaub H, Schenck S, Brügger B, Ringler P, Müller SA, Rammner B, Gräter F, Hub JS, De Groot BL, Mieskes G, Moriyama Y, Klingauf J, Grubmüller H, Heuser J, et al. (2006) Molecular anatomy of a trafficking organelle. Cell 127:831-846. CrossRef Medline

Tan PK, Waites C, Liu Y, Krantz DE, Edwards RH (1998) A leucine-based motif mediates the endocytosis of vesicular monoamine and acetylcholine transporters. J Biol Chem 273:17351-17360. CrossRef Medline

Traub LM, Ostrom JA, Kornfeld S (1993) Biochemical dissection of AP-1 recruitment onto Golgi membranes. J Cell Biol 123:561-573. CrossRef Medline

Vanden Berghe P, Klingauf J (2006) Synaptic vesicles in rat hippocampal boutons recycle to different pools in a use-dependent fashion. J Physiol 572:707-720. Medline

Vergarajauregui S, Puertollano R (2006) Two di-leucine motifs regulate trafficking of mucolipin-1 to lysosomes. Traffic 7:337-353. CrossRef Medline

Vinatier J, Herzog E, Plamont MA, Wojcik SM, Schmidt A, Brose N, Daviet L, El Mestikawy S, Giros B (2006) Interaction between the vesicular glutamate transporter type 1 and endophilin A1, a protein essential for endocytosis. J Neurochem 97:1111-1125. CrossRef Medline

Voglmaier SM, Edwards RH (2007) Do different endocytic pathways make different synaptic vesicles? Curr Opin Neurobiol 17:374-380. CrossRef Medline

Voglmaier SM, Kam K, Yang H, Fortin DL, Hua Z, Nicoll RA, Edwards RH (2006) Distinct endocytic pathways control the rate and extent of synaptic vesicle protein recycling. Neuron 51:71-84. CrossRef Medline

Weston MC, Nehring RB, Wojcik SM, Rosenmund C (2011) Interplay between VGLUT isoforms and endophilin A1 regulates neurotransmitter release and short-term plasticity. Neuron 69:1147-1159. CrossRef Medline

Wojcik SM, Rhee JS, Herzog E, Sigler A, Jahn R, Takamori S, Brose N, Rosenmund C (2004) An essential role for vesicular glutamate transporter 1 (VGLUT1) in postnatal development and control of quantal size. Proc Natl Acad Sci U S A 101:7158-7163. CrossRef Medline

Wucherpfennig T, Wilsch-Bräuninger M, González-Gaitán M (2003) Role of Drosophila Rab5 during endosomal trafficking at the synapse and evoked neurotransmitter release. J Cell Biol 161:609-624. CrossRef Medline

Zhang F, Gradinaru V, Adamantidis AR, Durand R, Airan RD, de Lecea L, Deisseroth K (2010) Optogenetic interrogation of neural circuits: technology for probing mammalian brain structures. Nat Protoc 5:439-456. CrossRef Medline

Zhao B, Wong AY, Murshid A, Bowie D, Presley JF, Bedford FK (2008) Identification of a novel di-leucine motif mediating $\mathrm{K}(+) / \mathrm{Cl}(-)$ cotransporter KCC2 constitutive endocytosis. Cell Signal 20:1769-1779. CrossRef Medline 